Supporting Information for

\title{
Functional Toxicogenomic Assessment of Triclosan in Human HepG2 Cells Using Genome-wide CRISPR-Cas9 Screen
}

$\mathrm{Pu}$ Xia $\dagger$, Xiaowei Zhang $\dagger^{*}$, Yuwei Xie $\dagger$, Miao Guan $\dagger$, Daniel L. Villeneuve + , Hongxia Yu†

$\dagger$ State Key Laboratory of Pollution Control \& Resource Reuse, School of the Environment, Nanjing University, Nanjing, P. R. China, 210023

fUnited States Environmental Protection Agency, Mid-Continent Ecology Division, Duluth, MN, USA.

\section{The file includes:}

59 pages

Text S1

Figure S1 to S9

Table S1 to S15

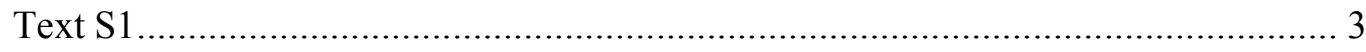

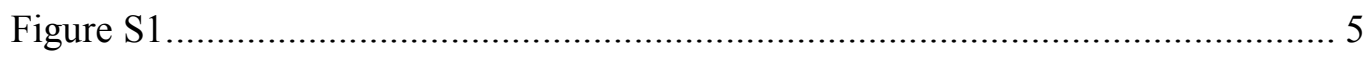

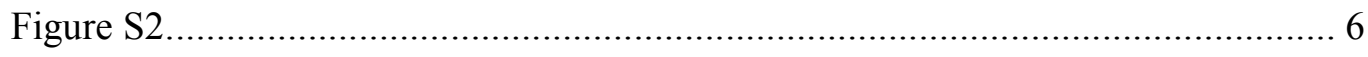

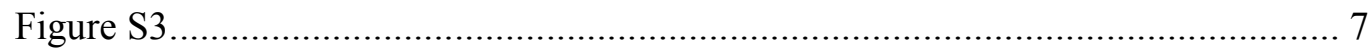

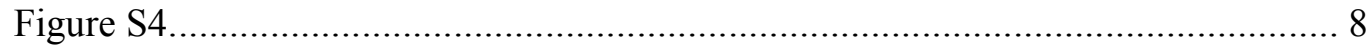

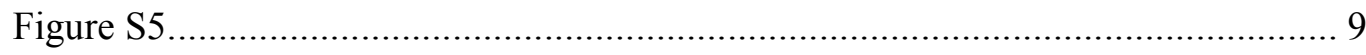




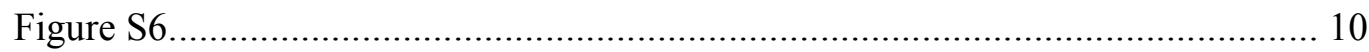

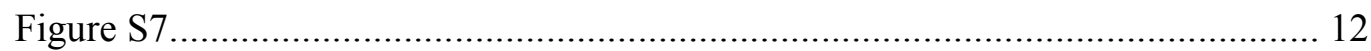

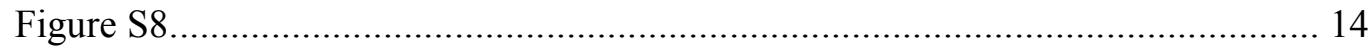

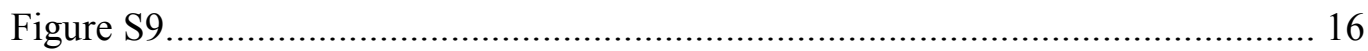

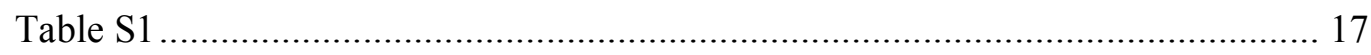

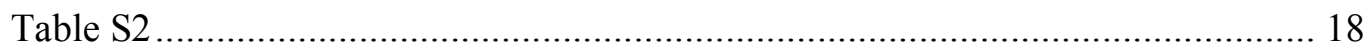

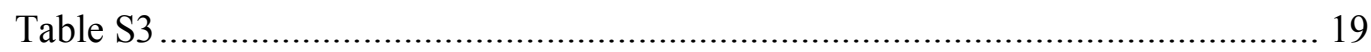

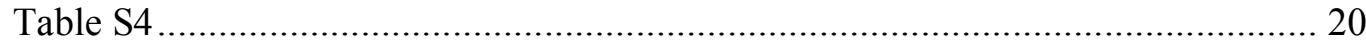

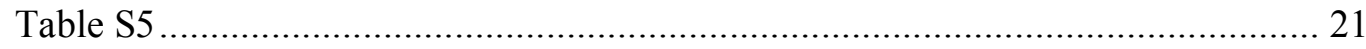

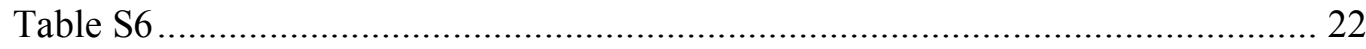

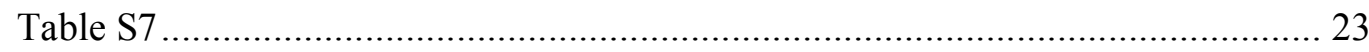

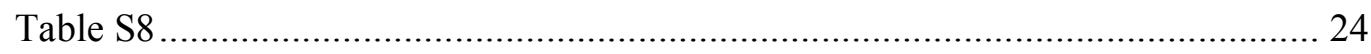

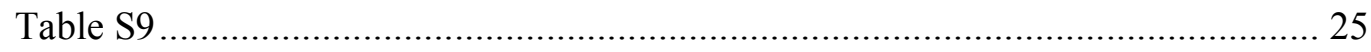

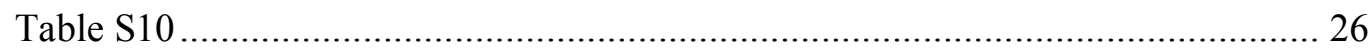

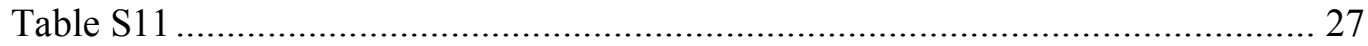

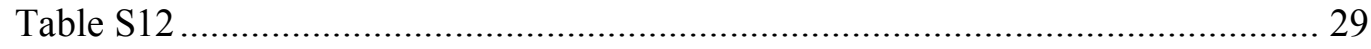

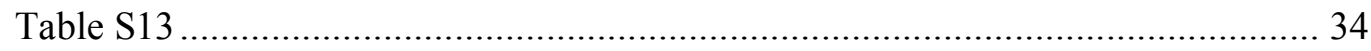

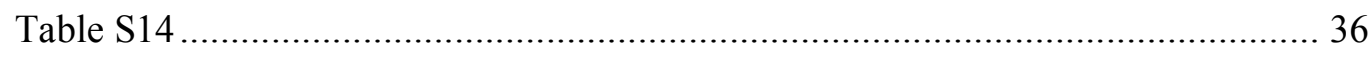

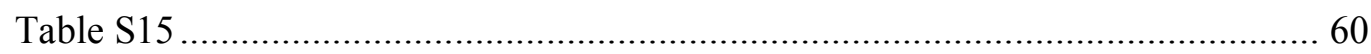


Text S1. Supplemental information that describes process of ToxCast data retrieval and use

ToxCast data for TCS was downloaded from the ToxCast database (https://www.epa.gov/chemical-research/toxicity-forecaster-toxcasttm-data). ToxCast includes 941 "gene-based" assays whose biological activity is related with specific genes (i.e., NR1I2), and 251 other assays targeting apical cellular phenotype (i.e., cellular viability). To compare with CRISPR-Cas9 functional genomic profile consisting of resistant/sensitive genes, 177 "gene-based" assays testing TCS with AC50 (half-maximal activity) $<10^{6}$ were selected. The scope of assays evaluation was further narrowed to 33 "gene-based HepG2" assays using HepG2 cells linked with 30 gene endpoints (i.e, Attagene platform measuring transcription factor activity of PPARG in HepG2 cells) using the same HepG2 cells to test TCS as the CRISPR-Cas9 screen conducted here. This step excluded "non-cell-based" assays (i.e., Novascreen platform testing receptor binding activity) and "non-HepG2-based" assays (i.e., Odyssey Thera platform using HEK293T cells). The gene targets from ToxCast "gene-based" assays were compared with gene-disease annotations from DisGeNET, which would reveal the number of genes from ToxCast "gene-based HepG2" assays annotated to disease terms. In some cases, genes may be tested in multiple assays, which result in multiple AC50 values for that gene. Here we assigned a calculated AC50 value for each gene. For instance, there were two "gene-based HepG2" assays (ATG_PPRE_CIS_up assay and ATG_PPARg_TRANS_up assay) in ToxCast whose endpoints are linked with PPARG, which have two AC50 values, 9.39 and $9.68 \mu \mathrm{M}$, 
respectively. The two AC50 values were averaged and then log-transformed, resulting a calculated AC50 value, 0.98 , for $P P A R G$.

Finally, we compared enriched disease terms between genes from ToxCast "gene-based HepG2" assays and genes from the whole ToxCast 941 "gene-based" assays. A Fisher's exact test was used and a p-value of 0.1 was used to determine a significant difference. Take obesity as instance, the R code for Fisher's exact test is as following:

\#\#\# Number of genes from "gene-based HepG2" assays annotated to obesity term a $1<-19$

\#\#\# Number of genes from "gene-based HepG2" assays not annotated to obesity term a $2<-(30-a 1)$ \#\#\# Number of whole ToxCast genes annotated to obesity term b1 $<-165$

\#\#\# Number of whole ToxCast genes not annotated to obesity term b2 $<-364-b 1$

\#\#\#Construction of $2 \times 2$ contingency table

Convictions <-matrix(c(a1-1, b1, a2, b2),

$$
\text { nrow }=2 \text {, }
$$

dimnames =list(c("My List", "Genome"),

c("In Pathway", "Not In Pathway"))) 
\#\#\# Fisher's exact test

fisher.test(Convictions)

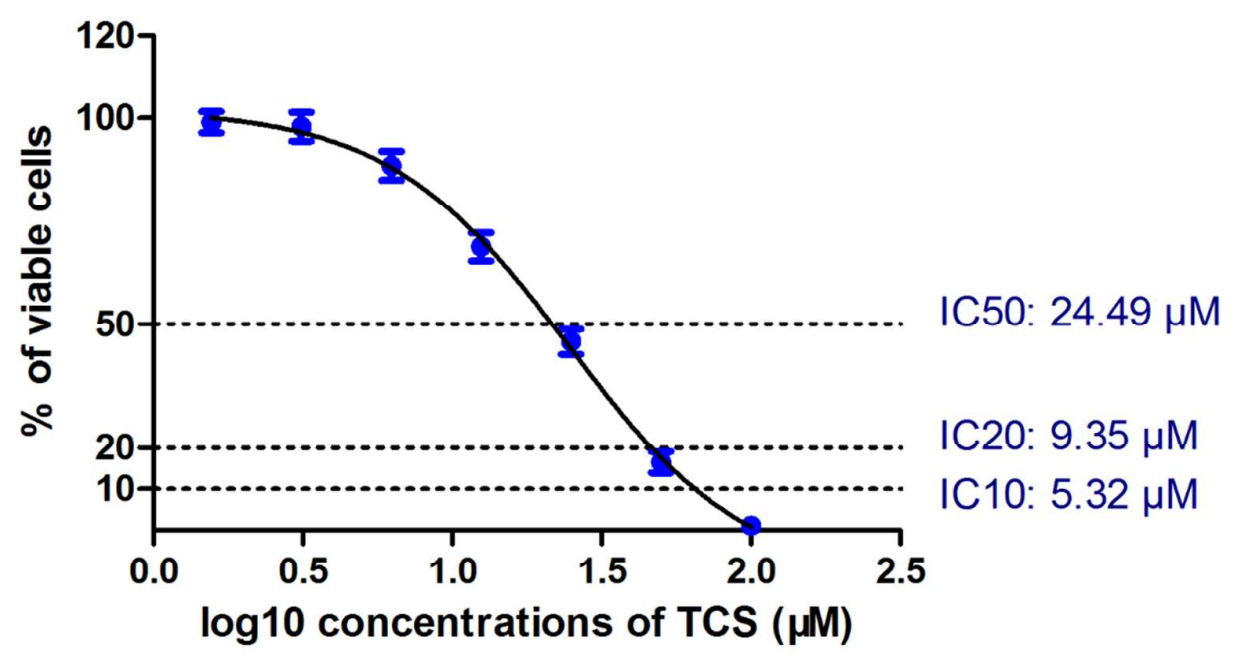

Figure S1. Cytotoxicity of TCS to HepG2 cell lines. Data represented are mean \pm SD of three identical experiments made in three replicate. 


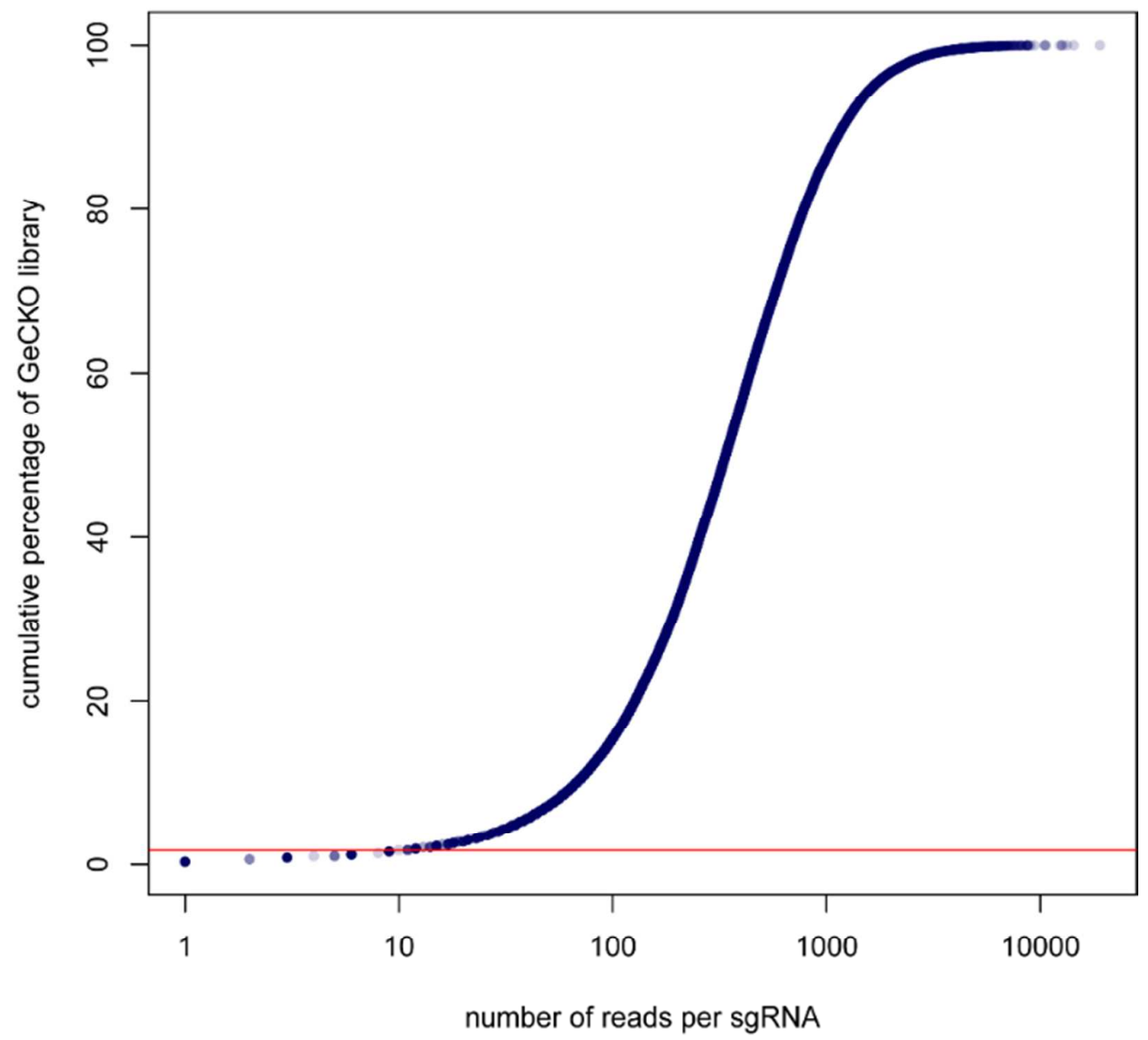

Figure S2. Cumulative distribution of the reads per sgRNA in GeCKO library. Each dot is a single sgRNA. Dots below the red line indicate that less than $3 \%$ sgRNAs are covered by less than 10 reads. 


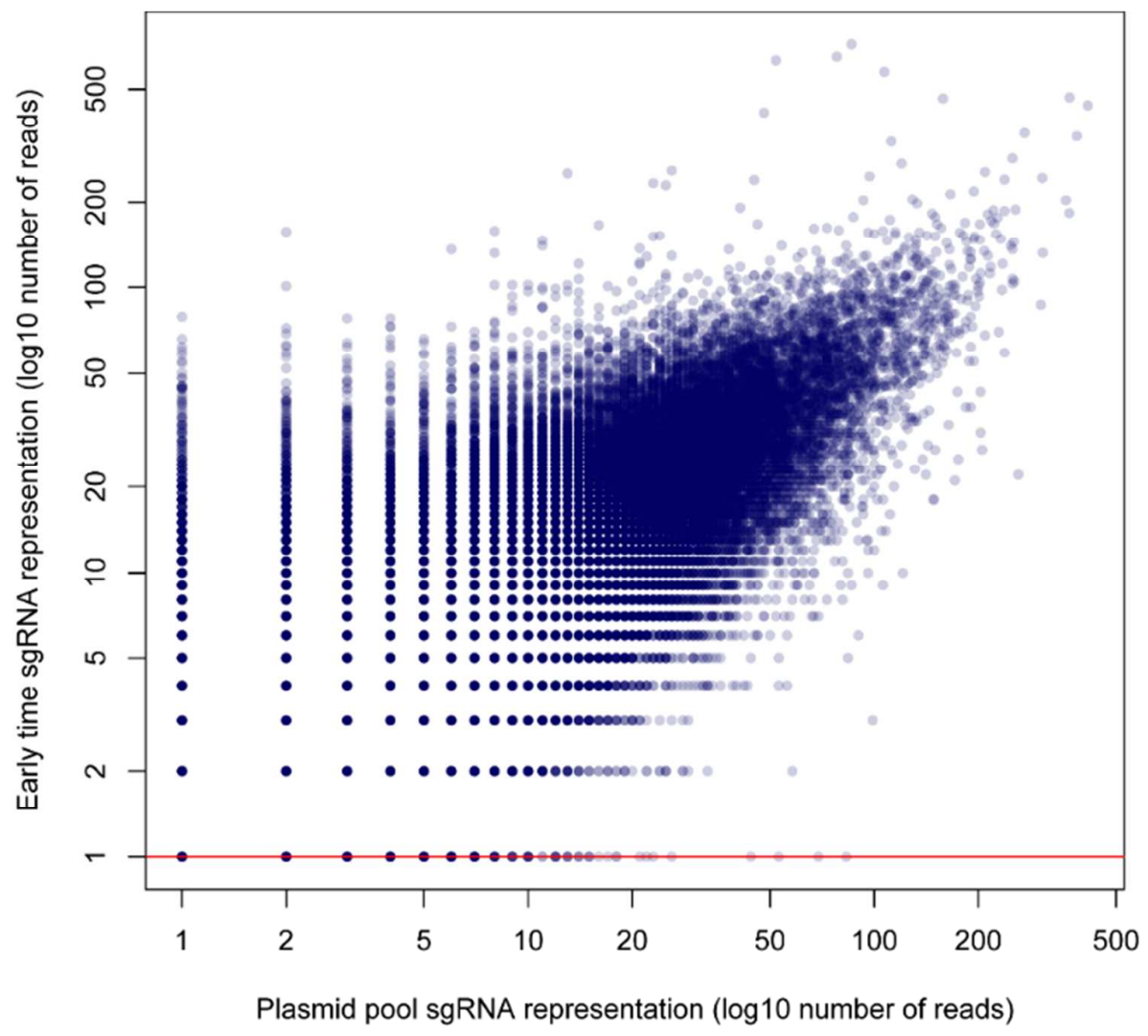

Figure S3. Comparison of sgRNA representation between the GeCKO plasmids pool and transduced cells. Dots below the red lines indicate that less than $0.17 \%$ of sgRNA were present at less than 10 reads. 


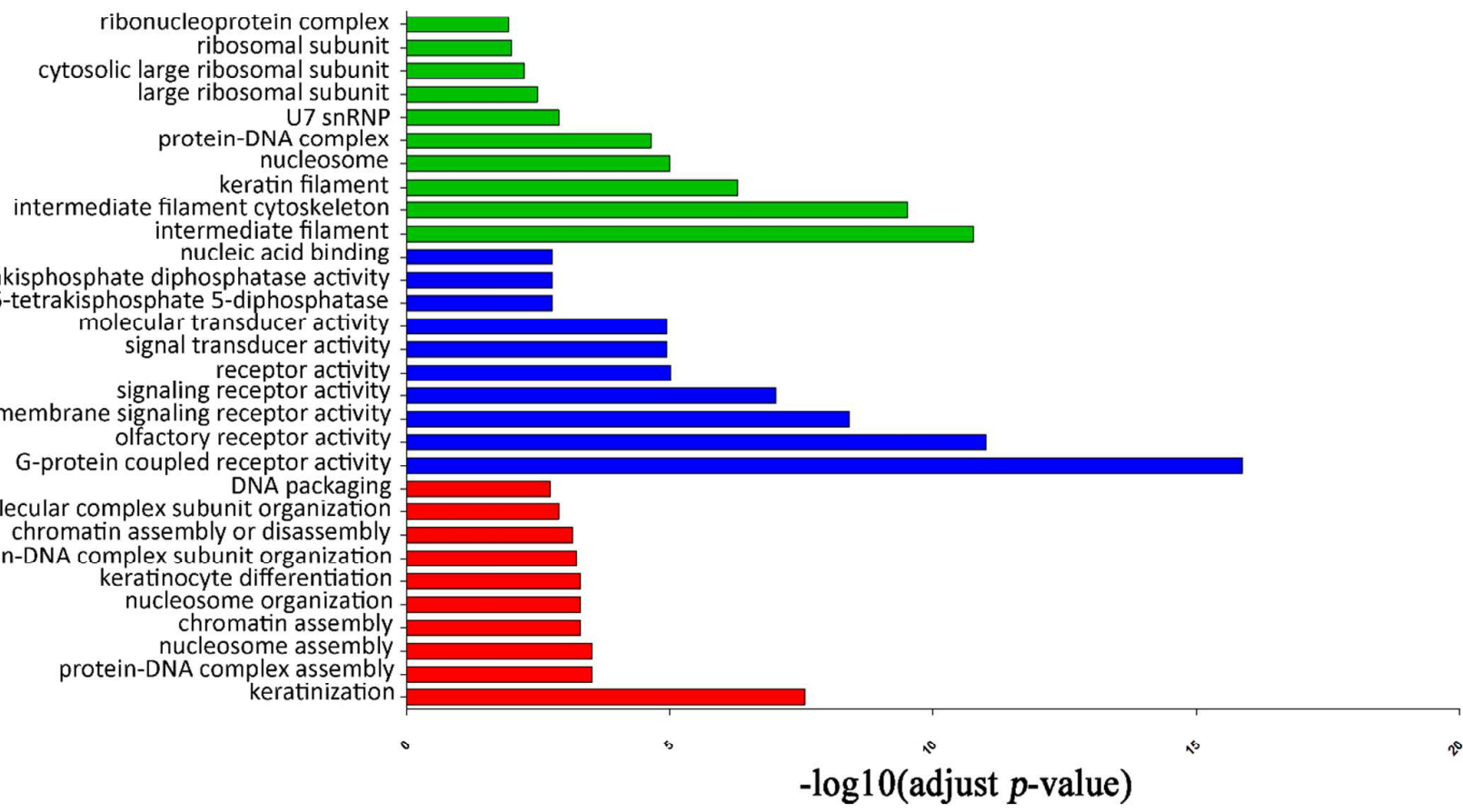

Figure S4. Gene Ontology (GO) terms enriched among the genes lost after transduction. Red, blue and green bars are GO terms of biological process (BP), molecular function (MF) and cellular component (CC), respectively. 
(A)

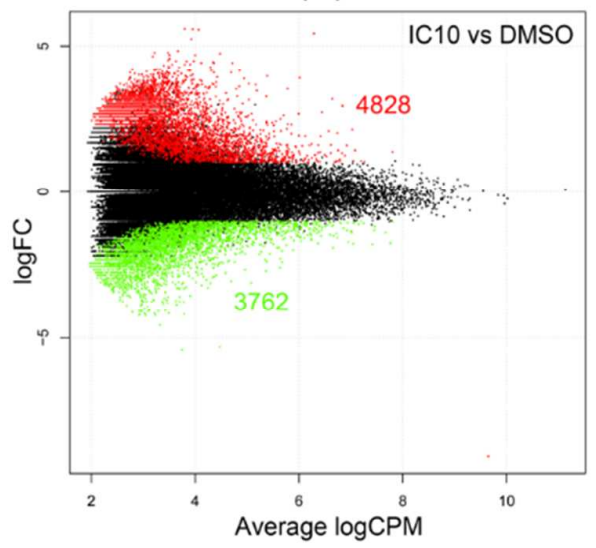

(C)

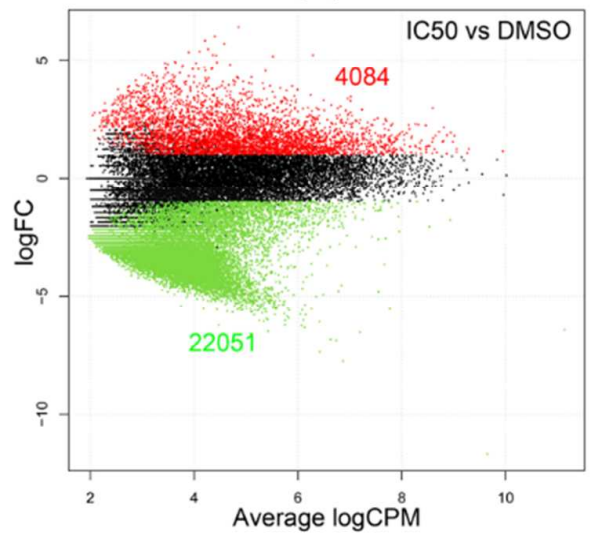

(B)

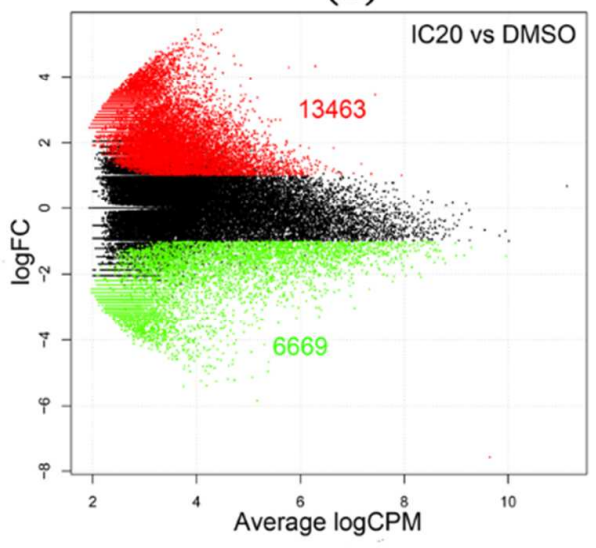

(D)

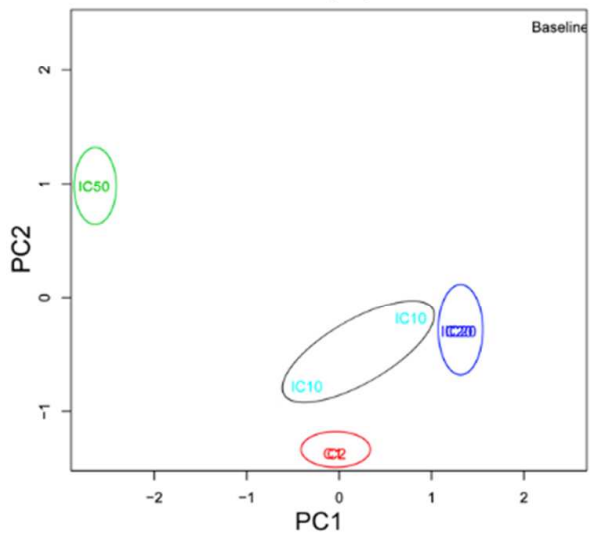

Figure S5. Analysis of sgRNA representation in each treatment group compared with vehicle control (DMSO). (A), (B) and (C) show significant overrepresentation (red dots) or underrepresentation (green dots) of sgRNAs $(\mathrm{FDR}<0.05)$ in IC10, IC20 and IC50, respectively; (D) shows the principle component analysis of each treatment and vehicle control group with two replicate. "Baseline" means the abundance of sgRNAs at Day 0 for control group. Groups inside red, light blue, dark blue and green circles mean vehicle control, IC10, IC20 and IC50, respectively. 
(A)

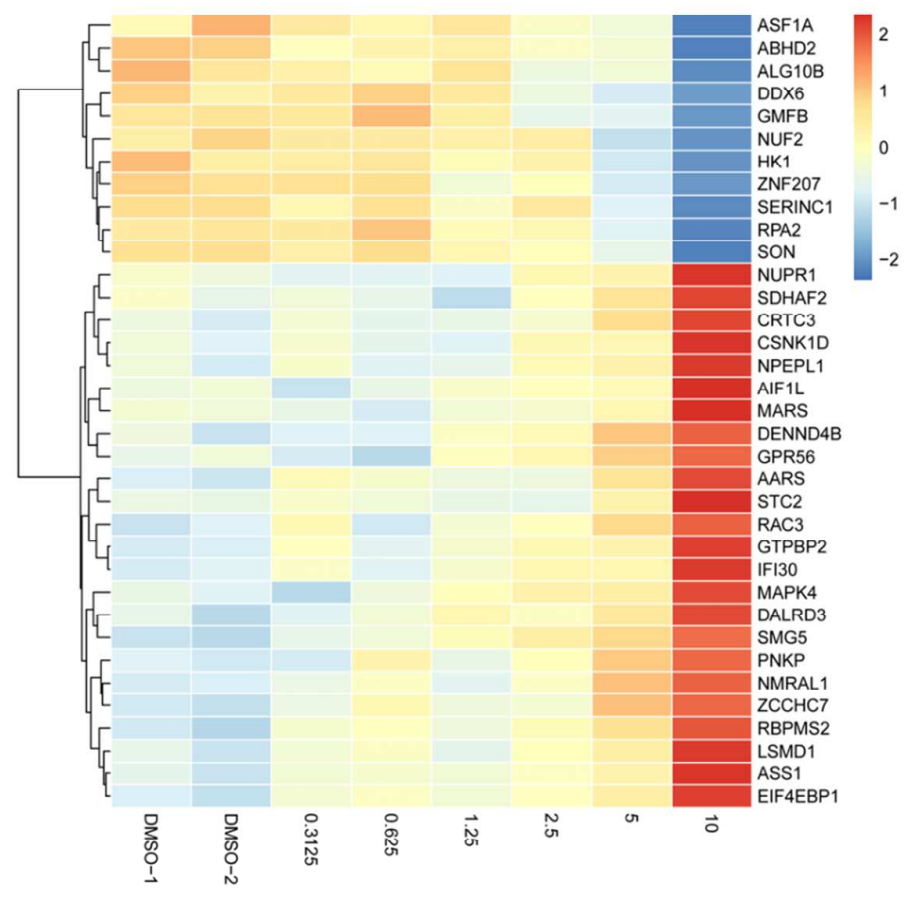

(B)

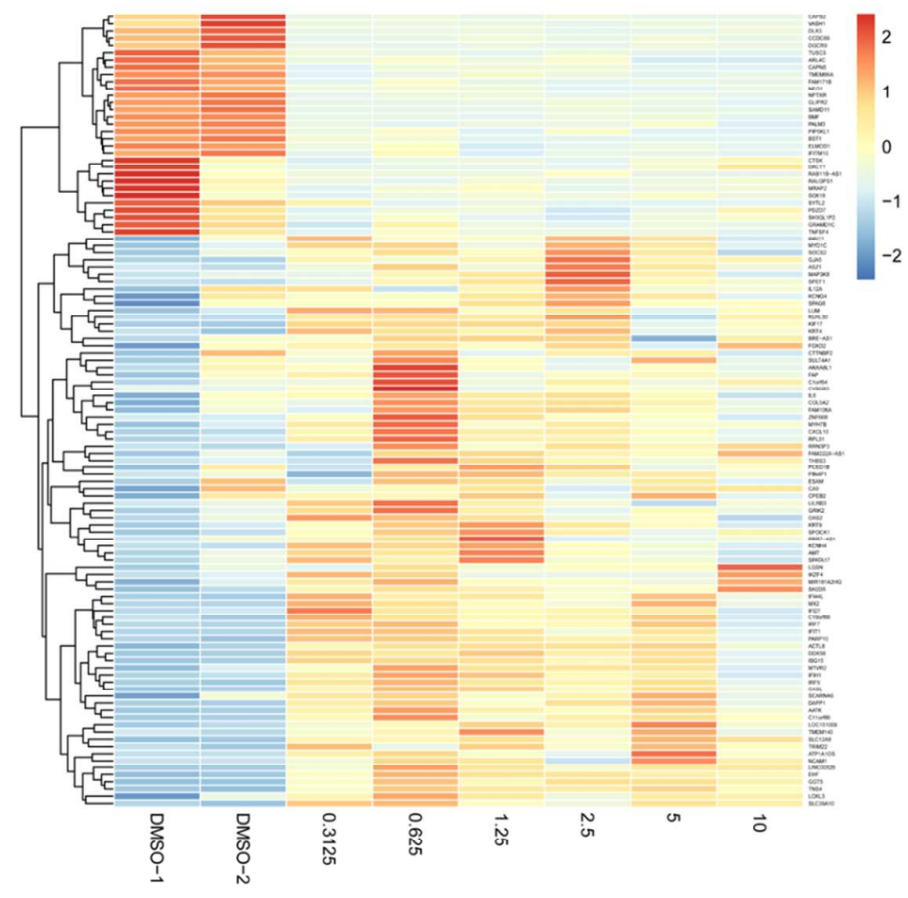

Figure S6. (A) Heatmap of expression of genes with significantly dose-dependent performance (adjusted p-value $<0.2$ ) of TCS transcriptomics in HepG2 cells. (B) Heatmap of expression of genes consistently altered $(\log 2$ fold changes $>1.5$ or $<-1.5)$ in TCS transcriptomics in HepG2 cells. 
The expressions were scaled by rows. The right columns are gene symbols, and the numbers in bottom rows are TCS concentrations of treatment. 
(A)

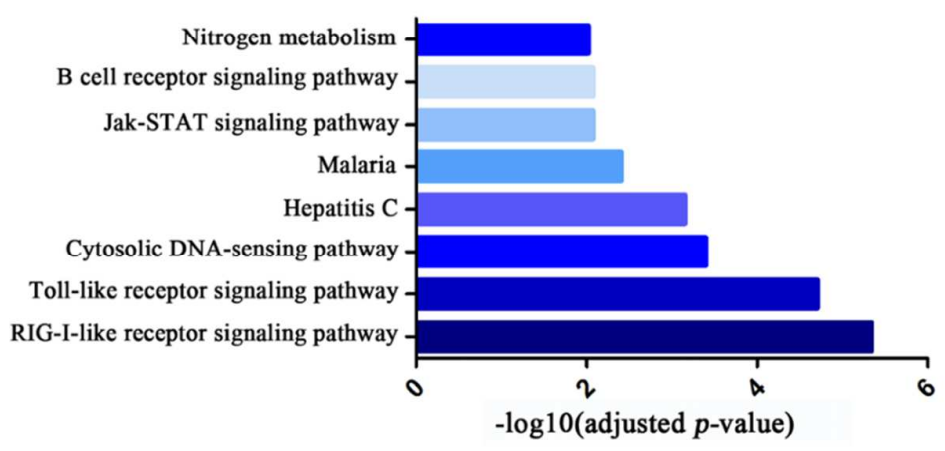

(B)

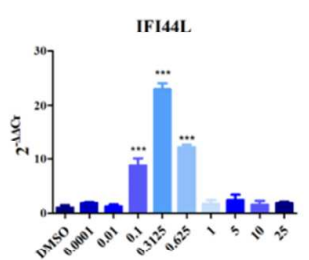

IRF9

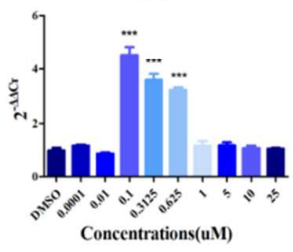

IFIT1

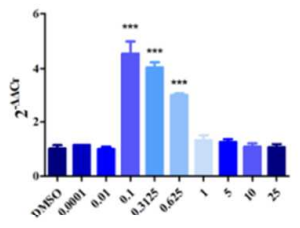

ISG15

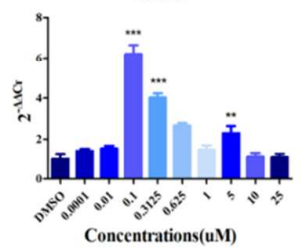

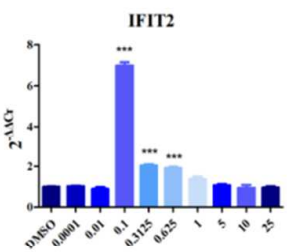

KRT7

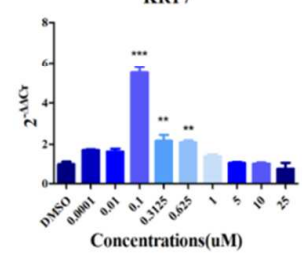

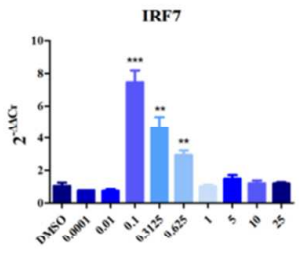

OASL

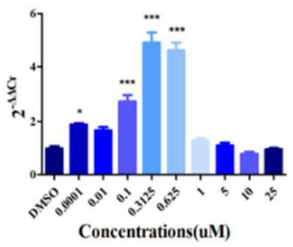

Figure S7. Transcriptome analysis of low doses of TCS. (A) The significantly enriched KEGG pathways associated with the DEGs and (B) qPCR validation of selected DEGs. 
(A)

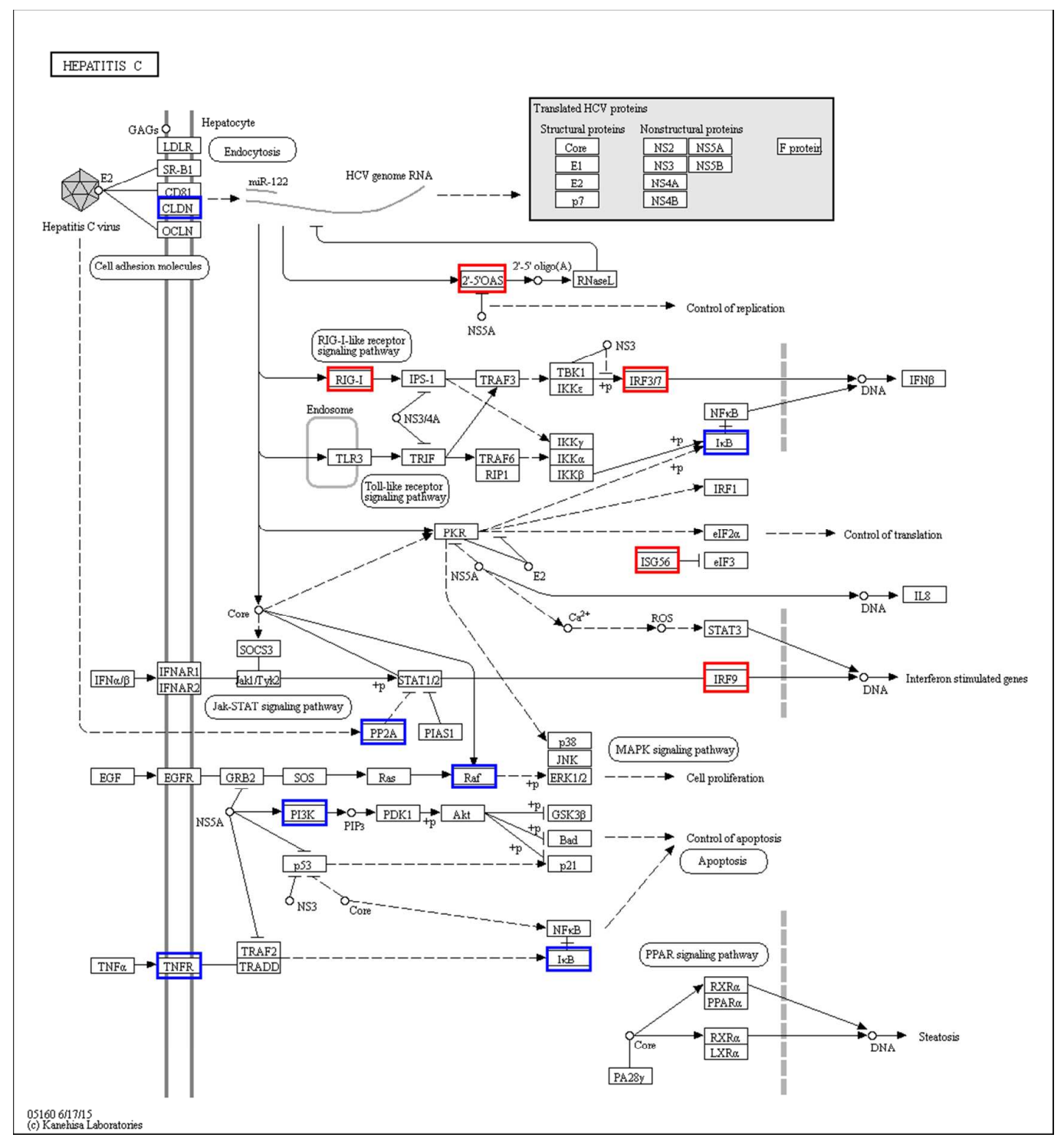


(B)

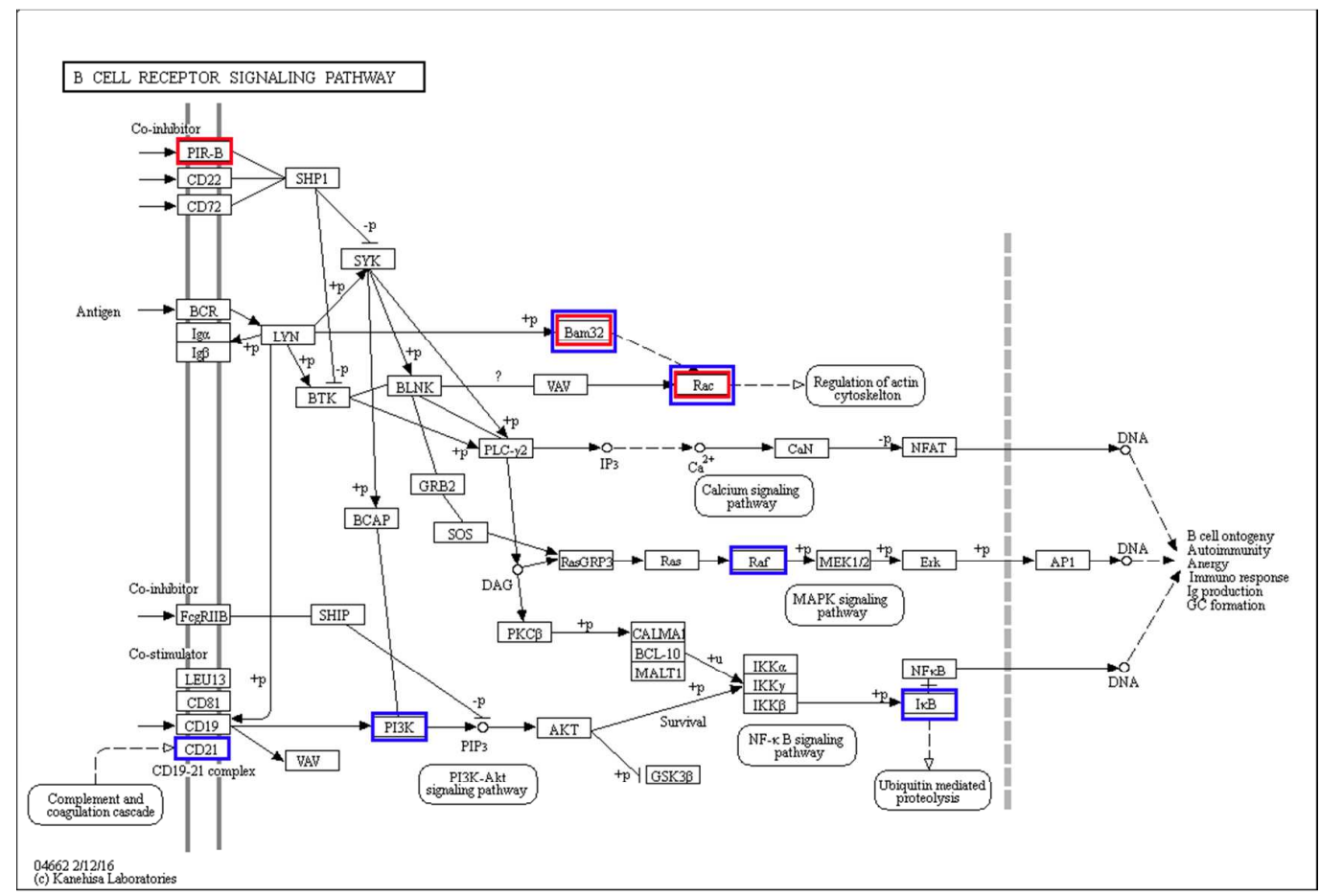

(C)

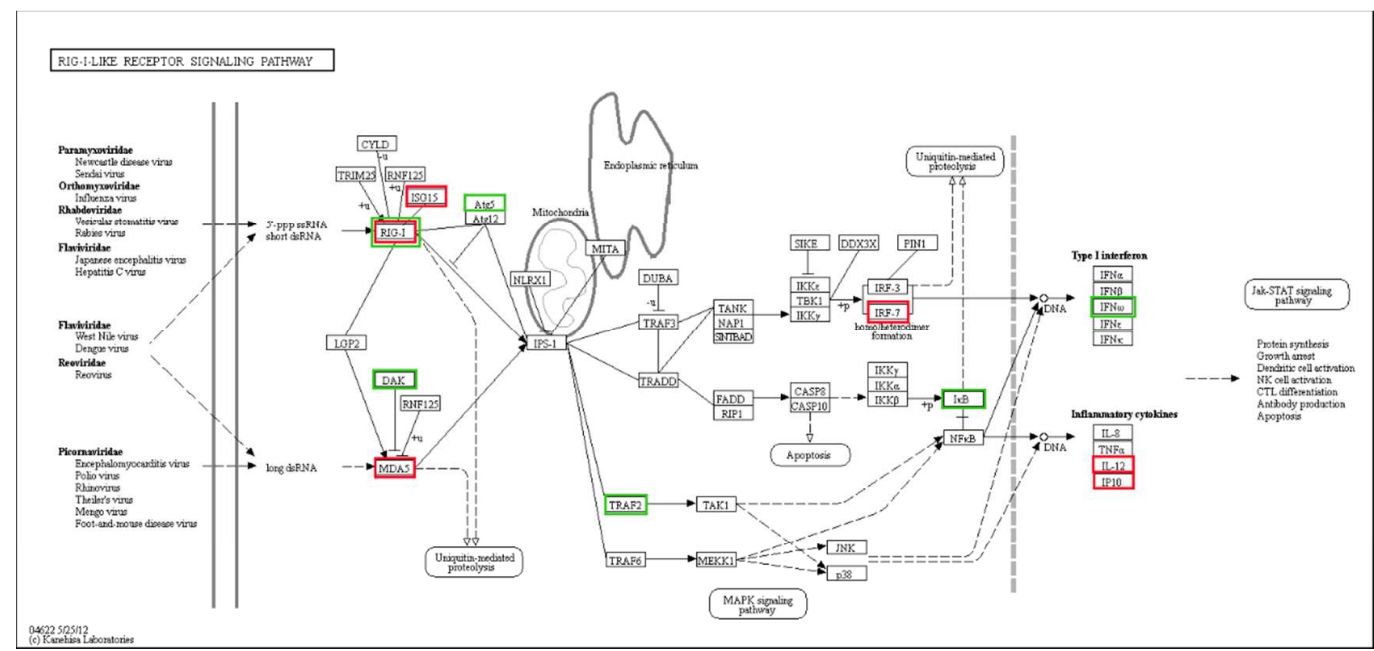

Figure S8. Common KEGG pathways significantly enriched by transcriptomic DEGs and CRISPR-Cas9 sensitive genes (IC10 and IC20). (A) Hepatitis C pathway; (B) B 
cell receptor signaling pathway; (C) RIG-I-like receptor signaling pathway. The red, blue and green rectangles mean enriched transcriptomic DEGs, CRISPR-Cas9 IC10 sensitive genes and CRISPR-Cas9 IC20 sensitive genes, respectively. 


\section{IC20 sensitive genes IC10 sensitive genes}

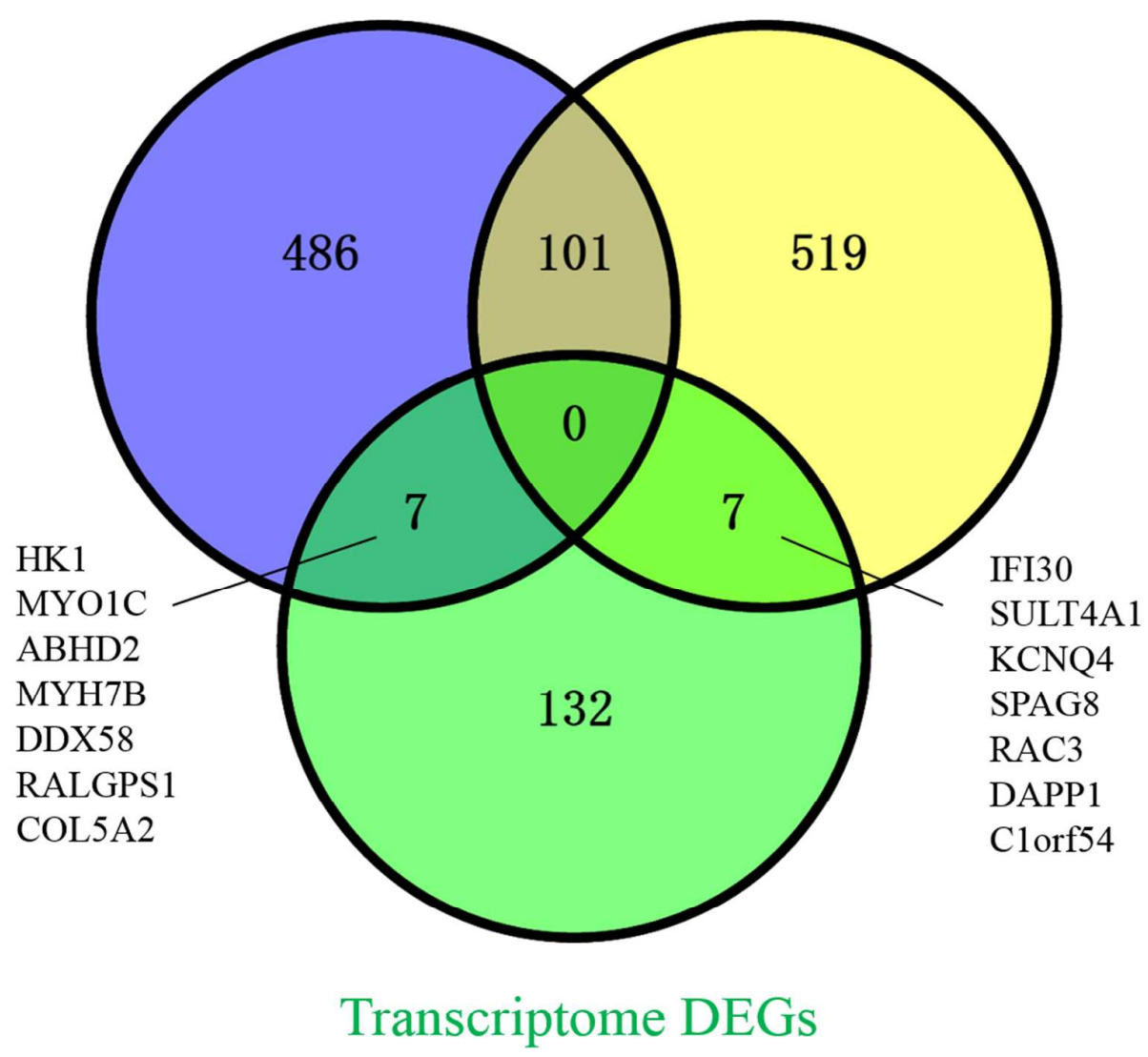

Figure S9. Comparison of genes between transcriptomic DEGs and CRISPR-Cas9 sensitive genes (IC10 and IC20). 
Table S1. Primers sequences to amplify lentiCRISPR sgRNAs for the first PCR

\begin{tabular}{l|l}
\hline Primers & Sequences \\
\hline $\mathbf{F 1}$ & AATGGACTATCATATGCTTACCGTAACTTGAAAGTATTTCG \\
\hline $\mathbf{R 1}$ & CTTTAGTTTGTATGTCTGTTGCTATTATGTCTACTATTCTTTCC \\
\hline
\end{tabular}

*The PCR conditions included initial heat-denaturing step at $98^{\circ} \mathrm{C}$ for $2 \mathrm{~min}, 20$

cycles of $98^{\circ} \mathrm{C}$ for $10 \mathrm{~s}, 60^{\circ} \mathrm{C}$ for $20 \mathrm{~s}$ and $72^{\circ} \mathrm{C}$ for $30 \mathrm{~s}$, and a final extension step at $72^{\circ} \mathrm{C}$ for 3 min on SureCycler 8800 (Agilent technologies, Santa Clara, CA, US). 
Table S2. Primers sequences to amplify lentiCRISPR sgRNAs for the second PCR

\begin{tabular}{cc}
\hline Primers & Sequences \\
\hline F2-A & CCATCTCATCCCTGCGTGTCTCCGACTCAG ATCATC GATTCTTGTGGAAAGGACGAAACACCG \\
\hline F2-B & CCATCTCATCCCTGCGTGTCTCCGACTCAG ATCAGATC GATTCTTGTGGAAAGGACGAAACACCG \\
\hline F2-C & CCATCTCATCCCTGCGTGTCTCCGACTCAGAAGAGGATTCGATTCTTGTGGAAAGGACGAAACACCG \\
\hline F2-D & CCATCTCATCCCTGCGTGTCTCCGACTCAGAGGACGCACTGTGATTCTTGTGGAAAGGACGAAACACCG \\
\hline F2-E & CCATCTCATCCCTGCGTGTCTCCGACTCAG CAGAGC GATTCTTGTGGAAAGGACGAAACACCG \\
\hline F2-F & CCATCTCATCCCTGCGTGTCTCCGACTCAG ATCTGAGC GATTCTTGTGGAAAGGACGAAACACCG \\
\hline F2-G & CCATCTCATCCCTGCGTGTCTCCGACTCAG ATAGGTTATA GATTCTTGTGGAAAGGACGAAACACCG \\
\hline F2-H & CCATCTCATCCCTGCGTGTCTCCGACTCAGACAGCTAGCTTGGATTCTTGTGGAAAGGACGAAACACCG \\
\hline R2 & CCACTACGCCTCCGCTTTCCTCTCTATGGGCAGTCGGTGATTCTACTATTCTTTCCCCTGCACTGT
\end{tabular}

*Due to the sequencing length limitation of Ion Torrent Proton, we modified the reverse primers to make the length of second PCR products less than 200 base pairs. The sequences in red represent barcodes of each sequencing library. 
Table S3. Oligo sequences for CRISPR-Cas9 plasmids construction for individual gene knockout

\begin{tabular}{lll}
\hline Gene symbols & Oligo sequences & Off-target score (OS) \\
\hline FTO & AGCTTCGCGCTCTCGTTCCT & 56.7368 \\
$M A P 2 K 3$ & CCGTGCGCATGTTGATGTCC & 27.6316 \\
\hline
\end{tabular}


Table S4. Primers of selected transcriptome differentially expressed genes for qPCR validation

\begin{tabular}{lll}
\hline Gene symbol & Sense primer & Antisense primer \\
\hline IFI44 & GTATAGCATATGTGGCCTTGCTTACT & ATGACCCGGCTTTGAGAAGTC \\
IFIT1 & GCAGCCAAGTTTTACCGAAG & GCCCTATCTGGTGATGCAGT \\
$I F I T 2$ & CGAACAGCTGAGAATTGCAC & CAAGTTCCAGGTGAAATGGC \\
$I R F 7$ & TACCATCTACCTGGGCTTCG & AGGGTTCCAGCTTCACCA \\
$I R F 9$ & CCCGAAAACTCCGGAACTG & CAGCACACTCCGGGAAACT \\
$I S G 15$ & GGTGGACAAATGCGACGAA & ATGCTGGTGGAGGCCCTTA \\
KRT7 & CTCTGTGATGAATTCCACTGGTG & CCCATGGTTCCCCCGA \\
OASL & CCATCACGGTCACCATTGTG & ACCGCAGGCCTTGATCAG \\
\hline
\end{tabular}


Table S5. Top 20 IC50 resistant genes identified from CRISPR-Cas9 functional genomic screening on triclosan

\begin{tabular}{|c|c|c|c|}
\hline Gene & Gene rank & p-value & p-value rank \\
\hline C17orf98 & 1 & 0.0001 & 3 \\
\hline MAP2K3 & 2 & 0.0001 & 2 \\
\hline FTO & 3 & 0.0001 & 1 \\
\hline FTSJD2 & 4 & 0.0003 & 4 \\
\hline $\mathrm{CCDC} 48$ & 5 & 0.001 & 10 \\
\hline SLC10A7 & 6 & 0.0013 & 16 \\
\hline C11orf63 & 7 & 0.0003 & 6 \\
\hline AARSD 1 & 8 & 0.001 & 12 \\
\hline LDHAL6A & 9 & 0.0003 & 5 \\
\hline POGZ & 10 & 0.0014 & 19 \\
\hline KIAA1407 & 11 & 0.0005 & 7 \\
\hline PNLDC1 & 12 & 0.0005 & 8 \\
\hline FAM47E & 13 & 0.0022 & 29 \\
\hline TBX15 & 14 & 0.0018 & 24 \\
\hline SHMT1 & 15 & 0.001 & 11 \\
\hline PDPR & 16 & 0.0009 & 9 \\
\hline ORAI1 & 17 & 0.0012 & 14 \\
\hline GKN1 & 18 & 0.0027 & 35 \\
\hline EMR1 & 19 & 0.0014 & 17 \\
\hline BTBD9 & 20 & 0.0035 & 41 \\
\hline
\end{tabular}


Table S6. Top 20 IC20 resistant genes identified from CRISPR-Cas9 functional genomic screening on triclosan

\begin{tabular}{|c|c|c|c|}
\hline Gene & Gene rank & p-value & p-value rank \\
\hline MUC4 & 1 & 0.0002 & 5 \\
\hline PLEKHB2 & 2 & 0.0002 & 4 \\
\hline ARGLU1 & 3 & 0.0001 & 1 \\
\hline KRTAP10-10 & 4 & 0.0001 & 2 \\
\hline SRC & 5 & 0.0003 & 8 \\
\hline ANKRD2 & 6 & 0.0008 & 11 \\
\hline PAN3 & 7 & 0.0003 & 7 \\
\hline EBAG9 & 8 & 0.0001 & 3 \\
\hline SDPR & 9 & 0.0008 & 12 \\
\hline MCM7 & 10 & 0.0008 & 13 \\
\hline CD83 & 11 & 0.0013 & 20 \\
\hline TCERG1L & 12 & 0.0003 & 6 \\
\hline DENND5B & 13 & 0.0005 & 9 \\
\hline TRIM55 & 14 & 0.0017 & 26 \\
\hline AEN & 15 & 0.0006 & 10 \\
\hline MPZ & 16 & 0.0016 & 25 \\
\hline BIK & 17 & 0.0023 & 34 \\
\hline CYBASC3 & 18 & 0.0026 & 40 \\
\hline UBE4B & 19 & 0.0024 & 36 \\
\hline TUBGCP2 & 20 & 0.0024 & 35 \\
\hline
\end{tabular}


Table S7. Top 20 IC10 resistant genes identified from CRISPR-Cas9 functional genomic screening on triclosan

\begin{tabular}{|c|c|c|c|}
\hline$\overline{\text { Gene }}$ & Gene rank & p-value & p-value rank \\
\hline GPC1 & 1 & 0.0002 & 4 \\
\hline DLC1 & 2 & 0.0001 & 3 \\
\hline CACHD1 & 3 & 0.0001 & 1 \\
\hline ENDOU & 4 & 0.0004 & 5 \\
\hline CLCF1 & 5 & 0.0011 & 14 \\
\hline SLC9B1 & 6 & 0.0011 & 15 \\
\hline S1PR3 & 7 & 0.0001 & 2 \\
\hline PSMB2 & 8 & 0.0012 & 16 \\
\hline SAMSN1 & 9 & 0.0023 & 27 \\
\hline TCF4 & 10 & 0.0033 & 39 \\
\hline BCHE & 11 & 0.0006 & 7 \\
\hline OR13A1 & 12 & 0.0006 & 6 \\
\hline HSD17B12 & 13 & 0.0007 & 8 \\
\hline RNF212 & 14 & 0.0009 & 12 \\
\hline OR10H3 & 15 & 0.0008 & 9 \\
\hline CACNG1 & 16 & 0.0008 & 10 \\
\hline CCDC51 & 17 & 0.0025 & 32 \\
\hline RAB11FIP5 & 18 & 0.0025 & 30 \\
\hline TMEM104 & 19 & 0.0009 & 11 \\
\hline IGSF8 & 20 & 0.0033 & 40 \\
\hline
\end{tabular}


Table S8. Top 20 IC50 sensitive genes identified from CRISPR-Cas9 functional genomic screening on triclosan

\begin{tabular}{|c|c|c|c|}
\hline Gene & Gene rank & p-value & p-value rank \\
\hline NOTCH2 & 1 & 0.0003 & 4 \\
\hline EXOC1 & 2 & 0.0002 & 1 \\
\hline DTX2 & 3 & 0.0003 & 3 \\
\hline SLC5A10 & 4 & 0.0008 & 8 \\
\hline SQRDL & 5 & 0.0008 & 9 \\
\hline RPA2 & 6 & 0.0008 & 10 \\
\hline ATP1A3 & 7 & 0.001 & 14 \\
\hline PNPLA8 & 8 & 0.0002 & 2 \\
\hline COL6A3 & 9 & 0.0012 & 17 \\
\hline CARD14 & 10 & 0.0003 & 5 \\
\hline ZNF317 & 11 & 0.0013 & 20 \\
\hline CCNY & 12 & 0.0014 & 21 \\
\hline SIGLEC8 & 13 & 0.0005 & 7 \\
\hline CMKLR1 & 14 & 0.0004 & 6 \\
\hline PSKH1 & 15 & 0.0018 & 30 \\
\hline SCAF1 & 16 & 0.0023 & 35 \\
\hline RAD51AP1 & 17 & 0.0009 & 12 \\
\hline STEAP3 & 18 & 0.0025 & 37 \\
\hline C2orf65 & 19 & 0.001 & 13 \\
\hline CHODL & 20 & 0.0024 & 36 \\
\hline
\end{tabular}


Table S9. Top 20 IC20 sensitive genes identified from CRISPR-Cas9 functional genomic screening on triclosan

\begin{tabular}{|c|c|c|c|}
\hline$\overline{\text { Gene }}$ & Gene rank & p-value & p-value rank \\
\hline ARHGAP12 & 1 & 0.0001 & 1 \\
\hline РТВР3 & 2 & 0.0012 & 9 \\
\hline TUBA8 & 3 & 0.0015 & 13 \\
\hline DAG1 & 4 & 0.0013 & 10 \\
\hline HS2ST1 & 5 & 0.0003 & 2 \\
\hline IFT27 & 6 & 0.0005 & 3 \\
\hline ETV3L & 7 & 0.0025 & 30 \\
\hline CDKL4 & 8 & 0.001 & 5 \\
\hline NMU & 9 & 0.0023 & 23 \\
\hline CELSR2 & 10 & 0.0024 & 25 \\
\hline TRAPPC 12 & 11 & 0.0009 & 4 \\
\hline ZNF317 & 12 & 0.003 & 35 \\
\hline KDM5C & 13 & 0.0032 & 38 \\
\hline ELMOD2 & 14 & 0.0014 & 12 \\
\hline SFI1 & 15 & 0.0034 & 40 \\
\hline PHKG1 & 16 & 0.0036 & 41 \\
\hline KRT73 & 17 & 0.001 & 6 \\
\hline HIST1H4D & 18 & 0.0011 & 8 \\
\hline CPNE3 & 19 & 0.0017 & 16 \\
\hline CPN2 & 20 & 0.0011 & 7 \\
\hline
\end{tabular}


Table S10. Top 20 IC10 sensitive genes identified from CRISPR-Cas9 functional genomic screening on triclosan

\begin{tabular}{|c|c|c|c|}
\hline$\overline{\text { Gene }}$ & Gene rank & p-value & p-value rank \\
\hline IFI30 & 1 & 0.0001 & 1 \\
\hline SQRDL & 2 & 0.0007 & 7 \\
\hline LTBP3 & 3 & 0.0008 & 8 \\
\hline FAM181A & 4 & 0.0003 & 2 \\
\hline ABRA & 5 & 0.001 & 9 \\
\hline C18orf54 & 6 & 0.0003 & 3 \\
\hline PRSS3 & 7 & 0.002 & 22 \\
\hline CRABP2 & 8 & 0.0015 & 15 \\
\hline GUCY2F & 9 & 0.0004 & 4 \\
\hline INHBE & 10 & 0.0005 & 5 \\
\hline RARS2 & 11 & 0.0006 & 6 \\
\hline BRD9 & 12 & 0.0018 & 19 \\
\hline ZNF365 & 13 & 0.0019 & 20 \\
\hline C1orf61 & 14 & 0.0021 & 24 \\
\hline TBC1D2 & 15 & 0.002 & 23 \\
\hline TTLL12 & 16 & 0.001 & 10 \\
\hline CELSR2 & 17 & 0.0023 & 25 \\
\hline KCNK2 & 18 & 0.0012 & 11 \\
\hline AJUBA & 19 & 0.0027 & 34 \\
\hline DEDD & 20 & 0.0013 & 13 \\
\hline
\end{tabular}


Table S11. Gene-Disease association of CRISPR-Cas9 resistant/sensitive genes identified by using GeneAnalytics. (A) Disease terms scores of IC50 resistant genes; (B) Disease terms scores of IC50 sensitive genes; (C) Disease terms scores of IC20 resistant genes; (D) Disease terms scores of IC10 resistant genes

(A)

\begin{tabular}{ll}
\hline Score & Disease terms \\
\hline 15.29 & Breast Cancer \\
\hline 14.94 & Influenza \\
\hline
\end{tabular}

(B)

\begin{tabular}{ll}
\hline Score & Disease terms \\
\hline 12.52 & Osteopontin-mediated Events \\
\hline 10.99 & G-protein Signaling_K-RAS Regulation Pathway \\
\hline 10.03 & EGFR1 Signaling Pathway \\
\hline 9.23 & Spliceosome \\
\hline 8.82 & PAK Pathway \\
\hline
\end{tabular}

(C)

\begin{tabular}{ll}
\hline Score & Disease terms \\
\hline 17.92 & Breast Cancer \\
\hline 16.01 & Obesity \\
\hline
\end{tabular}

(D) 


\begin{tabular}{ll}
\hline Score & Disease terms \\
\hline 20.64 & Colorectal Cancer \\
\hline 15.13 & Breast Cancer \\
\hline 15.03 & Malaria \\
\hline
\end{tabular}

*Disease terms scores of IC20 sensitive genes and IC10 sensitive genes were not exhibited, due to no medium scores were obtained by disease terms of the two groups of genes. 
Table S12. Consistently differential expression genes of transcriptome analysis of low doses TCS. (A) Consistently up-regulated genes; (B) Consistently down-regulated genes

(A)

\begin{tabular}{|c|c|c|c|c|c|c|}
\hline Genes & 10 & 5 & 2.5 & 1.25 & 0.625 & 0.3125 \\
\hline AATK & 1.868853 & 2.007438 & 1.745126 & 1.818239 & 1.884051 & 1.984244 \\
\hline ACTL8 & 1.927555 & 2.090689 & 2.180698 & 2.132054 & 1.655345 & 2.023035 \\
\hline AMT & 1.690516 & 2.225618 & 2.988053 & 3.909662 & 2.877737 & 3.642763 \\
\hline ANXA8L1 & 2.275478 & 2.547546 & 1.988053 & 2.646627 & 3.578177 & 2.794766 \\
\hline ASZ1 & 2.012444 & 2.547546 & 3.40309 & 1.646627 & 2.462699 & 1.794766 \\
\hline ATP1A1OS & 2.275478 & 3.032973 & 1.40309 & 1.909662 & 2.199665 & 2.472838 \\
\hline BIRC7 & 1.690516 & 2.225618 & 2.573015 & 1.909662 & 1.462699 & 2.642763 \\
\hline BRE-AS1 & 2.690516 & 2.650423 & 2.725018 & 2.646627 & 2.199665 & 2.057801 \\
\hline C11orf86 & 1.896377 & 1.804668 & 1.835722 & 1.406424 & 1.788752 & 1.757105 \\
\hline C19orf66 & 0.990076 & 2.319594 & 2.094968 & 1.872187 & 1.792007 & 2.714913 \\
\hline C1 orf54 & 3.012444 & 1.810581 & 2.725018 & 1.909662 & 3.047662 & 2.057801 \\
\hline CA9 & 3.275478 & 2.810581 & 1.988053 & 2.909662 & 2.199665 & 2.472838 \\
\hline COL5A2 & 1.649874 & 1.610282 & 1.988053 & 1.8272 & 1.73018 & 1.432196 \\
\hline CPEB2 & 2.175943 & 2.810581 & 1.888517 & 2.810126 & 1.948126 & 2.543227 \\
\hline CTTNBP2 & 2.275478 & 3.225618 & 3.40309 & 2.324699 & 3.462699 & 3.057801 \\
\hline CXCL10 & 3.938443 & 4.51102 & 5.046946 & 4.909662 & 5.287128 & 4.93227 \\
\hline CYB5R3 & 1.690516 & 1.810581 & 2.40309 & 0.324699 & 3.784628 & 2.472838 \\
\hline DAPP1 & 2.860441 & 3.869474 & 3.795408 & 3.025139 & 3.401299 & 3.642763 \\
\hline DDX58 & 1.714822 & 2.310607 & 2.288342 & 2.519313 & 1.827861 & 2.603321 \\
\hline
\end{tabular}




\begin{tabular}{|c|c|c|c|c|c|c|}
\hline EHF & 3.342593 & 3.032973 & 3.363919 & 3.324699 & 3.114776 & 2.969264 \\
\hline ESAM & 2.275478 & 3.032973 & 2.40309 & 3.324699 & 3.047662 & 1.472838 \\
\hline FAM106A & 1.828019 & 1.711045 & 2.251087 & 2.090234 & 1.948126 & 1.320835 \\
\hline FAM222A-AS1 & 2.629115 & 1.640656 & 1.90559 & 2.132054 & 1.685092 & 0.69523 \\
\hline FAP & 2.175943 & 2.151618 & 2.168625 & 1.703211 & 2.685092 & 2.472838 \\
\hline FOXD2 & 2.912908 & 1.448011 & 2.518567 & 2.061665 & 1.877737 & 2.347307 \\
\hline GGT5 & 2.168563 & 1.910116 & 2.087588 & 1.726798 & 1.685092 & 1.709877 \\
\hline GJA5 & 2.690516 & 2.225618 & 3.210445 & 2.324699 & 1.462699 & 2.472838 \\
\hline GRIK2 & 1.583601 & 1.526788 & 1.087588 & 1.746163 & 1.940747 & 2.124915 \\
\hline IFI27 & 1.218447 & 2.225618 & 2.224753 & 2.06836 & 1.959267 & 3.123389 \\
\hline IFI44L & 3.860441 & 4.769939 & 4.027581 & 4.597718 & 3.9652 & 5.116694 \\
\hline IFIH1 & 1.095772 & 1.623495 & 1.66937 & 2.040058 & 1.718132 & 1.914436 \\
\hline IFIT1 & 1.45855 & 2.052008 & 1.572652 & 2.152933 & 1.88833 & 2.532909 \\
\hline IKZF4 & 4.012444 & 1.810581 & 1.988053 & 1.909662 & 2.877737 & 3.642763 \\
\hline IL12A & 2.105553 & 1.640656 & 2.725018 & 2.061665 & 0.614703 & 2.472838 \\
\hline IL6 & 2.690516 & 3.313081 & 3.862522 & 3.646627 & 3.632624 & 3.560301 \\
\hline IRF7 & 1.127783 & 2.084526 & 1.769141 & 1.644834 & 1.792373 & 2.291668 \\
\hline IRF9 & 1.646572 & 2.144005 & 2.067573 & 2.164659 & 2.021783 & 2.316822 \\
\hline ISG15 & 1.771204 & 2.3319 & 2.167874 & 2.418075 & 2.024304 & 2.629303 \\
\hline KCNH4 & 1.275478 & 1.547546 & 1.988053 & 2.784131 & 2.047662 & 2.794766 \\
\hline KCNQ4 & 1.690516 & 1.810581 & 2.210445 & 1.909662 & 1.199665 & 1.794766 \\
\hline KIF17 & 2.275478 & 1.225618 & 2.40309 & 2.324699 & 1.877737 & 2.472838 \\
\hline KLHL30 & 2.275478 & 0.225618 & 2.573015 & 2.025139 & 1.685092 & 2.173278 \\
\hline KRT4 & 2.275478 & 1.225618 & 2.988053 & 2.324699 & 2.199665 & 3.057801 \\
\hline KRT9 & 1.690516 & 2.225618 & 2.725018 & 3.132054 & 2.462699 & 2.472838 \\
\hline
\end{tabular}




\begin{tabular}{|c|c|c|c|c|c|c|}
\hline LGSN & 3.690516 & 2.225618 & 2.40309 & 2.132054 & 2.337169 & 1.794766 \\
\hline LILRB3 & 2.012444 & -0.77438 & 1.725018 & 2.324699 & 2.784628 & 2.93227 \\
\hline LINC00526 & 2.497871 & 2.032973 & 1.725018 & 2.132054 & 2.047662 & 1.794766 \\
\hline LOC151009 & 1.95355 & 2.427252 & 1.988053 & 2.212225 & 1.363164 & 1.735872 \\
\hline LOXL3 & 3.082833 & 2.68505 & 2.309981 & 2.784131 & 2.685092 & 2.794766 \\
\hline LUM & 2.012444 & 1.00989 & 2.04052 & 1.581039 & 1.73018 & 2.369002 \\
\hline MAP3K8 & 1.275478 & 2.68505 & 3.795408 & 2.909662 & 2.199665 & 1.794766 \\
\hline MIR181A2HG & 4.012444 & 2.547546 & 2.988053 & 2.909662 & 3.199665 & 3.472838 \\
\hline MTVR2 & 1.690516 & 2.225618 & 2.40309 & 2.324699 & 2.199665 & 2.057801 \\
\hline MX2 & 3.012444 & 4.032973 & 2.988053 & 3.324699 & 3.401299 & 4.280193 \\
\hline МYH7B & 2.275478 & 1.810581 & 2.40309 & 1.909662 & 2.462699 & 2.472838 \\
\hline MYO1C & 1.690516 & 2.225618 & 2.725018 & 1.646627 & 2.047662 & 2.642763 \\
\hline NCAM1 & 2.690516 & 2.926058 & 0.40309 & 2.494624 & 2.199665 & 1.794766 \\
\hline OAS2 & 0.690516 & 2.225618 & 1.988053 & 2.909662 & 2.685092 & 3.472838 \\
\hline OASL & 2.244368 & 2.592989 & 2.368043 & 2.744777 & 2.389711 & 2.521074 \\
\hline PARP10 & 1.89215 & 2.60413 & 2.695872 & 2.810126 & 2.600203 & 3.238373 \\
\hline PCED1B & 2.690516 & 1.810581 & 2.988053 & 3.132054 & 2.199665 & 1.472838 \\
\hline PIN4P1 & 1.690516 & 1.810581 & 1.988053 & 2.324699 & 1.877737 & 1.854075 \\
\hline PRR7-AS1 & 3.012444 & 2.225618 & 1.40309 & 4.494624 & 3.337169 & 3.472838 \\
\hline RPL31 & 1.583601 & 1.418263 & 1.917663 & 1.839272 & 2.114776 & 2.057801 \\
\hline RRN3P3 & 2.912908 & 1.962584 & 2.518567 & 1.739737 & 2.462699 & 0.887876 \\
\hline SCARNA6 & 1.990076 & 2.407822 & 2.194503 & 1.909662 & 1.932185 & 2.387108 \\
\hline SH2D5 & 2.73491 & 1.547546 & 1.651018 & 1.848261 & 1.578177 & 1.93227 \\
\hline SLC12A8 & 2.327946 & 2.055693 & 1.625482 & 1.962129 & 1.292774 & 1.887876 \\
\hline SLC39A10 & 2.275478 & 1.953539 & 1.38037 & 1.610102 & 1.831933 & 2.355481 \\
\hline
\end{tabular}




\begin{tabular}{lllllll}
\hline SOCS2 & 1.690516 & 3.395543 & 4.10353 & 3.132054 & 3.199665 & 3.280193 \\
\hline SPAG8 & 2.690516 & 2.225618 & 2.988053 & 2.646627 & 1.877737 & 2.472838 \\
\hline SPATA17 & 1.275478 & 1.810581 & 1.988053 & 3.025139 & 1.877737 & 2.93227 \\
\hline SPEF1 & 2.275478 & 2.225618 & 3.40309 & 2.646627 & 1.877737 & 1.472838 \\
\hline SPOCK1 & 2.373326 & 1.263093 & 2.109359 & 2.584566 & 1.915212 & 2.00627 \\
\hline SULT4A1 & 1.597406 & 2.179815 & 1.210445 & 1.646627 & 1.9652 & 1.560301 \\
\hline THBS3 & 2.690516 & 1.547546 & 2.210445 & 2.646627 & 2.685092 & 0.472838 \\
\hline TMEM140 & 2.012444 & 3.473546 & 2.210445 & 3.717017 & 2.337169 & 2.472838 \\
\hline TNS4 & 2.197476 & 2.170336 & 2.158379 & 2.073548 & 1.866849 & 1.885161 \\
\hline TRIM22 & 2.275478 & 2.547546 & 1.818128 & 2.547092 & 0.877737 & 2.794766 \\
\hline ZNF668 & 0.690516 & 1.810581 & 1.988053 & 2.646627 & 2.877737 & 2.057801 \\
\hline
\end{tabular}

(B)

\begin{tabular}{lllllll}
\hline Genes & $\mathbf{1 0}$ & $\mathbf{5}$ & $\mathbf{2 . 5}$ & $\mathbf{1 . 2 5}$ & $\mathbf{0 . 6 2 5}$ & $\mathbf{0 . 3 1 2 5}$ \\
\hline ARL4C & -2.7018 & -3.1667 & -1.18187 & -1.74569 & -1.70723 & -1.59755 \\
BMF & -2.69031 & -1.93281 & -1.86225 & -3.05612 & -3.00058 & -2.40548 \\
BST1 & -1.89445 & -2.35934 & -2.18187 & -2.26026 & -1.70723 & -1.11212 \\
CAPN5 & -3.89445 & -3.35934 & -1.85994 & -1.93834 & -2.3853 & -4.11212 \\
CAPS2 & -3.11684 & -3.58174 & -2.40426 & -3.48266 & -2.92962 & -3.33452 \\
CCDC69 & -1.7018 & -1.58174 & -1.98923 & -1.48266 & -2.51458 & -2.33452 \\
CTSK & -0.81198 & -1.86184 & -2.68437 & -2.1778 & -2.62476 & -3.61462 \\
DACT1 & -0.30948 & -1.77438 & -2.59691 & -2.6753 & -3.12226 & -2.52716 \\
DGCR9 & -1.72452 & -1.77438 & -2.59691 & -2.09034 & -3.12226 & -1.9422 \\
DLX3 & -1.33696 & -2.91734 & -2.32483 & -1.81826 & -2.52826 & -2.08516 \\
ELMOD1 & -1.89445 & -1.77438 & -2.18187 & -3.26026 & -1.3853 & -1.52716 \\
\hline
\end{tabular}




\begin{tabular}{lllllll}
\hline FAM171B & -1.49006 & -1.77438 & -1.98393 & -1.76276 & -1.56587 & -1.80727 \\
GLIPR2 & -1.83305 & -1.71298 & -1.21358 & -1.6139 & -2.3239 & -1.59129 \\
GRAMD1C & -1.51112 & -1.71298 & -2.12047 & -1.6139 & -1.18639 & -3.05072 \\
IFITM10 & -1.88064 & -1.95126 & -1.65349 & -2.91631 & -1.09478 & -1.97967 \\
MRAP2 & -1.76892 & -2.49685 & -3.05634 & -1.39777 & -2.3593 & -3.57156 \\
NEO1 & -2.33923 & -5.38909 & -4.21162 & -2.12009 & -2.27754 & -2.14187 \\
NPTXR & -2.47941 & -2.41379 & -2.47733 & -1.84523 & -2.46211 & -2.69709 \\
PALM3 & -3.83305 & -1.29794 & -2.12047 & -2.19886 & -3.06086 & -2.05072 \\
PDZD7 & -0.47941 & -1.94431 & -2.76683 & -1.5233 & -1.97026 & -2.11212 \\
PIP5KL1 & -2.30948 & -2.77438 & -1.01195 & -2.6753 & -1.5373 & -1.52716 \\
RAB11B-AS1 & -2.06437 & -2.52927 & -1.3518 & -2.10826 & -2.55522 & -1.96012 \\
RALGPS1 & -1.89445 & -2.77438 & -3.18187 & -2.26026 & -4.70723 & -2.52716 \\
SAMD11 & -1.70977 & -1.98622 & -1.56548 & -2.07558 & -2.45696 & -1.96139 \\
SH3GL1P2 & -0.89445 & -1.77438 & -3.18187 & -2.26026 & -1.70723 & -2.11212 \\
SOX18 & -1.57252 & -1.55199 & -1.85994 & -1.6753 & -2.3853 & -2.52716 \\
SYTL2 & -2.89445 & -3.35934 & -3.18187 & -2.26026 & -3.70723 & -0.7902 \\
TMEM86A & -2.27296 & -4.05978 & -2.29735 & -1.63877 & -3.40767 & -4.81256 \\
\hline & -3.21637 & -2.68127 & -2.5038 & -2.58219 & -2.02915 & -3.43405 \\
VASH1 & -1.89445 & -2.35934 & -3.18187 & -2.26026 & -2.70723 & -2.11212 \\
\hline & & & & & & \\
\hline
\end{tabular}

Numbers in first row were concentrations of TCS used in transcriptome experiment.

Numbers in other rows are $\log 2$ fold change value of genes. 
Table S13. 22 disease terms (Score $>20$ ) associated with TCS retrieved from CTD databases

\begin{tabular}{|c|c|c|c|}
\hline Disease Name & Disease ID & Inference Score & Reference Count \\
\hline Reperfusion Injury & MESH:D015427 & 41.43 & 28 \\
\hline Breast Neoplasms & MESH:D001943 & 38.35 & 42 \\
\hline Carcinoma, Hepatocellular & MESH:D006528 & 33.58 & 27 \\
\hline Inflammation & MESH:D007249 & 31.05 & 37 \\
\hline Prostatic Neoplasms & MESH:D011471 & 29.9 & 49 \\
\hline Arthritis, Experimental & MESH:D001169 & 29.23 & 14 \\
\hline Infertility, Female & MESH:D007247 & 28.07 & 5 \\
\hline Arthritis, Rheumatoid & MESH:D001172 & 27.46 & 13 \\
\hline Obesity & MESH:D009765 & 27.31 & 13 \\
\hline Kidney Failure, Chronic & MESH:D007676 & 27.04 & 4 \\
\hline Heat Stroke & MESH:D018883 & 26.24 & 1 \\
\hline Colonic Neoplasms & MESH:D003110 & 25.91 & 16 \\
\hline
\end{tabular}




\begin{tabular}{llll}
\hline Diabetes Mellitus, Experimental & MESH:D003921 & 25.74 & 15 \\
Brain Ischemia & MESH:D002545 & 24.28 & 13 \\
Stomach Neoplasms & MESH:D013274 & 22.28 & 18 \\
Diabetes Mellitus, Type 2 & MESH:D003924 & 22.12 & 15 \\
HIV Wasting Syndrome & MESH:D019247 & 21.25 & 3 \\
Burns & MESH:D002056 & 21.23 & 4 \\
Thyroid Neoplasms & MESH:D013964 & 20.95 & 8 \\
Alzheimer Disease & MESH:D000544 & 20.41 & 20 \\
Drug-Induced Liver Injury & MESH:D056486 & 20.28 & 2 \\
\hline
\end{tabular}


Table S14. ToxCast Assays testing TCS

\begin{tabular}{|c|c|c|c|c|c|}
\hline Assay names & $\log 10(\mathrm{AC50})$ & Endpoints & AssayType & Platforms & CellType \\
\hline NVS_TR_hNET & -0.33 & SLC6A2 & binding reporter & Novascreen & NA \\
\hline TOX21_MMP_ratio_down & 0.15 & NA & membrane potential reporter & Tox21/NCGC & HepG2 \\
\hline NVS_ADME_hCYP2C19 & 0.26 & CYP2C19 & enzyme reporter & Novascreen & NA \\
\hline NHEERL_ZF_144hpf_TERATOSCORE_up & 0.42 & NA & NA & $\begin{array}{l}\text { National } \\
\text { Health and } \\
\text { Environmental } \\
\text { Effects } \\
\text { Research Lab - } \\
\text { Padilla Lab }\end{array}$ & $\begin{array}{l}\text { zebrafish } \\
\text { embryo }\end{array}$ \\
\hline ATG_PXRE_CIS_up & 0.51 & NR1I2 & inducible reporter & Attagene & HepG2 \\
\hline NVS_GPCR_rV1 & 0.53 & Avpr1a & binding reporter & Novascreen & NA \\
\hline ATG_NRF2_ARE_CIS_up & 0.54 & NFE2L2 & inducible reporter & Attagene & HepG2 \\
\hline ATG_PXR_TRANS_up & 0.61 & NR1I2 & inducible reporter & Attagene & HepG2 \\
\hline ATG_VDRE_CIS_up & 0.66 & VDR & inducible reporter & Attagene & HepG2 \\
\hline ATG_BRE_CIS_up & 0.67 & SMAD1 & inducible reporter & Attagene & HepG2 \\
\hline
\end{tabular}




\begin{tabular}{|c|c|c|c|c|c|}
\hline ATG_PBREM_CIS_up & 0.67 & NR1I3 & inducible reporter & Attagene & HepG2 \\
\hline BSK_hDFCGF_EGFR_down & 0.67 & EGFR & binding reporter & Bioseek & $\begin{array}{l}\text { foreskin } \\
\text { fibroblast }\end{array}$ \\
\hline BSK_hDFCGF_Proliferation_down & 0.69 & NA & viability reporter & Bioseek & $\begin{array}{l}\text { foreskin } \\
\text { fibroblast }\end{array}$ \\
\hline BSK_hDFCGF_CollagenIII_down & 0.69 & COL3A1 & binding reporter & Bioseek & $\begin{array}{l}\text { foreskin } \\
\text { fibroblast }\end{array}$ \\
\hline NVS_ENZ_hLck_Activator & 0.72 & LCK & enzyme reporter & Novascreen & NA \\
\hline BSK_hDFCGF_VCAM1_down & 0.72 & VCAM1 & binding reporter & Bioseek & $\begin{array}{l}\text { foreskin } \\
\text { fibroblast }\end{array}$ \\
\hline NVS_ADME_hCYP2C8 & 0.72 & CYP2C8 & enzyme reporter & Novascreen & NA \\
\hline BSK_LPS_PGE2_down & 0.74 & PTGER2 & binding reporter & Bioseek & $\begin{array}{l}\text { umbilical vein } \\
\text { endothelium and } \\
\text { peripheral blood } \\
\text { mononuclear } \\
\text { cells }\end{array}$ \\
\hline NVS_ADME_hCYP2C9 & 0.77 & CYP2C9 & enzyme reporter & Novascreen & NA \\
\hline ATG_RXRb_TRANS_up & 0.77 & RXRB & inducible reporter & Attagene & HepG2 \\
\hline
\end{tabular}




\begin{tabular}{|c|c|c|c|c|c|}
\hline NVS_MP_rPBR & 0.78 & Tspo & binding reporter & Novascreen & NA \\
\hline BSK_4H_VEGFRII_down & 0.81 & KDR & binding reporter & Bioseek & $\begin{array}{l}\text { umbilical vein } \\
\text { endothelium }\end{array}$ \\
\hline BSK_hDFCGF_PAI1_down & 0.82 & SERPINE1 & binding reporter & Bioseek & $\begin{array}{l}\text { foreskin } \\
\text { fibroblast }\end{array}$ \\
\hline NVS_ADME_hCYP1A2 & 0.82 & CYP1A2 & enzyme reporter & Novascreen & NA \\
\hline BSK_3C_Proliferation_down & 0.83 & NA & viability reporter & Bioseek & $\begin{array}{l}\text { umbilical vein } \\
\text { endothelium }\end{array}$ \\
\hline ATG_ISRE_CIS_dn & 0.83 & IRF1 & inducible reporter & Attagene & HepG2 \\
\hline BSK_hDFCGF_MCSF_down & 0.83 & CSF1 & binding reporter & Bioseek & $\begin{array}{l}\text { foreskin } \\
\text { fibroblast }\end{array}$ \\
\hline NVS_GPCR_hAdra2C & 0.83 & ADRA2C & binding reporter & Novascreen & NA \\
\hline BSK_SAg_Proliferation_down & 0.83 & NA & viability reporter & Bioseek & $\begin{array}{l}\text { umbilical vein } \\
\text { endothelium and } \\
\text { peripheral blood } \\
\text { mononuclear } \\
\text { cells }\end{array}$ \\
\hline NVS_NR_hPPARg & 0.85 & PPARG & binding reporter & Novascreen & NA \\
\hline
\end{tabular}




\begin{tabular}{|c|c|c|c|c|c|}
\hline BSK_3C_TissueFactor_down & 0.85 & F3 & binding reporter & Bioseek & $\begin{array}{l}\text { umbilical vein } \\
\text { endothelium }\end{array}$ \\
\hline ATG_CMV_CIS_up & 0.86 & NA & inducible reporter & Attagene & HepG2 \\
\hline BSK_3C_uPAR_down & 0.86 & PLAUR & binding reporter & Bioseek & $\begin{array}{l}\text { umbilical vein } \\
\text { endothelium }\end{array}$ \\
\hline BSK_CASM3C_IL6_down & 0.86 & IL6 & binding reporter & Bioseek & $\begin{array}{l}\text { coronary artery } \\
\text { smooth muscle } \\
\text { cells }\end{array}$ \\
\hline BSK_hDFCGF_IP10_down & 0.87 & CXCL10 & binding reporter & Bioseek & $\begin{array}{l}\text { foreskin } \\
\text { fibroblast }\end{array}$ \\
\hline ATG_TCF_b_cat_CIS_dn & 0.87 & LEF1|TCF7|TCF7L1|TCF7L2 & inducible reporter & Attagene & HepG2 \\
\hline BSK_3C_SRB_down & 0.87 & NA & viability reporter & Bioseek & $\begin{array}{l}\text { umbilical vein } \\
\text { endothelium }\end{array}$ \\
\hline APR_HepG2_CellCycleArrest_24h_dn & 0.88 & NA & morphology reporter & Apredica & HepG2 \\
\hline BSK_4H_MCP1_down & 0.89 & CCL2 & binding reporter & Bioseek & $\begin{array}{l}\text { umbilical vein } \\
\text { endothelium }\end{array}$ \\
\hline ATG_Sox_CIS_up & 0.89 & SOX1 & inducible reporter & Attagene & HepG2 \\
\hline BSK_hDFCGF_SRB_down & 0.89 & NA & viability reporter & Bioseek & foreskin \\
\hline
\end{tabular}




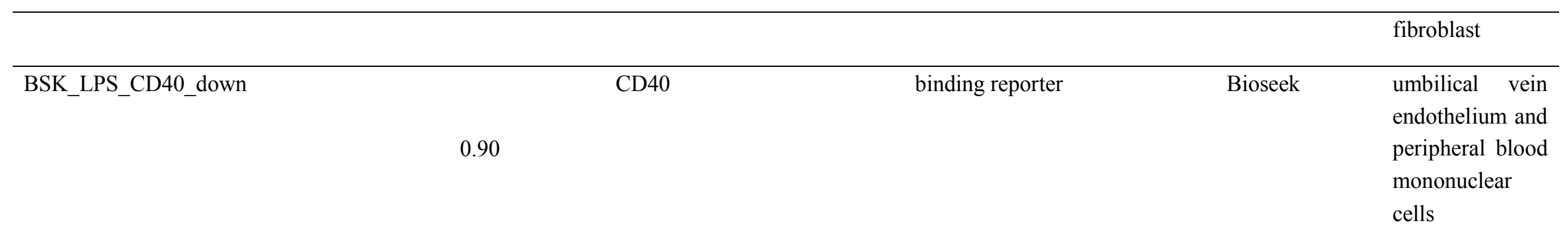

\begin{tabular}{|c|c|c|c|c|c|}
\hline APR_Hepat_DNATexture_1hr_dn & 0.90 & NA & conformation reporter & Apredica & hepatocyte \\
\hline BSK_SAg_IL8_down & 0.90 & CXCL8 & binding reporter & Bioseek & $\begin{array}{l}\text { umbilical vein } \\
\text { endothelium and } \\
\text { peripheral blood } \\
\text { mononuclear } \\
\text { cells }\end{array}$ \\
\hline
\end{tabular}

\begin{tabular}{|c|c|c|c|c|c|}
\hline ATG_AP_1_CIS_up & 0.91 & JUN|FOS & inducible reporter & Attagene & HepG2 \\
\hline ATG_EGR_CIS_up & 0.91 & EGR1 & inducible reporter & Attagene & HepG2 \\
\hline APR_HepG2_CellCycleArrest_72h_dn & 0.92 & NA & morphology reporter & Apredica & HepG2 \\
\hline NVS_ENZ_hBACE & 0.92 & BACE1 & enzyme reporter & Novascreen & NA \\
\hline BSK_SAg_MCP1_down & 0.92 & CCL2 & binding reporter & Bioseek & $\begin{array}{l}\text { umbilical vein } \\
\text { endothelium and } \\
\text { peripheral blood } \\
\text { mononuclear }\end{array}$ \\
\hline
\end{tabular}




\begin{tabular}{|c|c|c|c|c|c|}
\hline ATG_Pax6_CIS_up & 0.92 & PAX6 & inducible reporter & Attagene & HepG2 \\
\hline BSK_SAg_CD40_down & 0.93 & CD40 & binding reporter & Bioseek & $\begin{array}{l}\text { umbilical vein } \\
\text { endothelium and } \\
\text { peripheral blood } \\
\text { mononuclear } \\
\text { cells }\end{array}$ \\
\hline
\end{tabular}

\begin{tabular}{|c|c|c|c|c|c|}
\hline BSK_4H_Pselectin_down & 0.93 & SELP & binding reporter & Bioseek & $\begin{array}{l}\text { umbilical vein } \\
\text { endothelium }\end{array}$ \\
\hline ATG_Xbp1_CIS_up & 0.93 & XBP1 & inducible reporter & Attagene & HepG2 \\
\hline BSK_SAg_CD38_down & 0.94 & CD38 & binding reporter & Bioseek & $\begin{array}{l}\text { umbilical vein } \\
\text { endothelium and } \\
\text { peripheral blood } \\
\text { mononuclear } \\
\text { cells }\end{array}$ \\
\hline BSK_KF3CT_TGFb1_down & 0.94 & TGFB1 & binding reporter & Bioseek & $\begin{array}{l}\text { keratinocytes } \\
\text { and foreskin } \\
\text { fibroblasts }\end{array}$ \\
\hline BSK_hDFCGF_MMP1_down & 0.94 & MMP1 & binding reporter & Bioseek & $\begin{array}{l}\text { foreskin } \\
\text { fibroblast }\end{array}$ \\
\hline
\end{tabular}




\begin{tabular}{|c|c|c|c|c|c|}
\hline ATG_Oct_MLP_CIS_up & 0.94 & POU2F1 & inducible reporter & Attagene & HepG2 \\
\hline BSK_SAg_PBMCCytotoxicity_down & 0.94 & NA & viability reporter & Bioseek & $\begin{array}{l}\text { umbilical vein } \\
\text { endothelium and } \\
\text { peripheral blood } \\
\text { mononuclear } \\
\text { cells }\end{array}$ \\
\hline ATG_MRE_CIS_up & 0.94 & MTF1 & inducible reporter & Attagene & HepG2 \\
\hline BSK_hDFCGF_MIG_down & 0.95 & CXCL9 & binding reporter & Bioseek & $\begin{array}{l}\text { foreskin } \\
\text { fibroblast }\end{array}$ \\
\hline NVS_NR_hAR & 0.95 & $\mathrm{AR}$ & binding reporter & Novascreen & NA \\
\hline BSK_3C_Vis_down & 0.95 & NA & morphology reporter & Bioseek & $\begin{array}{l}\text { umbilical vein } \\
\text { endothelium }\end{array}$ \\
\hline BSK_SAg_Eselectin_down & 0.95 & SELE & binding reporter & Bioseek & $\begin{array}{l}\text { umbilical vein } \\
\text { endothelium and } \\
\text { peripheral blood } \\
\text { mononuclear } \\
\text { cells }\end{array}$ \\
\hline TOX21_TR_LUC_GH3_Antagonist & 0.96 & THRB|THRA & inducible reporter & Tox21/NCGC & GH3 \\
\hline ATG_Sp1_CIS_up & 0.96 & SP1 & inducible reporter & Attagene & HepG2 \\
\hline
\end{tabular}




\begin{tabular}{|c|c|c|c|c|c|}
\hline APR_HepG2_MitoMembPot_24h_dn & 0.96 & NA & membrane potential reporter & Apredica & HepG2 \\
\hline NVS_ENZ_rMAOAP & 0.96 & Maoa & enzyme reporter & Novascreen & NA \\
\hline NCCT_HEK293T_CellTiterGLO & 0.97 & NA & viability reporter & $\begin{array}{l}\text { NCCT's } \\
\text { Simmons Lab }\end{array}$ & HEK293T \\
\hline BSK_hDFCGF_TIMP1_down & 0.97 & TIMP1 & binding reporter & Bioseek & $\begin{array}{l}\text { foreskin } \\
\text { fibroblast }\end{array}$ \\
\hline ATG_PPRE_CIS_up & 0.97 & PPARA|PPARD|PPARG & inducible reporter & Attagene & HepG2 \\
\hline OT_AR_ARSRC1_0480 & 0.99 & $\mathrm{AR} \mid \mathrm{SRC}$ & binding reporter & Odyssey Thera & HEK293T \\
\hline APR_HepG2_MitoMembPot_72h_dn & 0.99 & NA & membrane potential reporter & Apredica & HepG2 \\
\hline BSK_KF3CT_MMP9_down & 0.99 & MMP9 & binding reporter & Bioseek & $\begin{array}{l}\text { keratinocytes } \\
\text { and foreskin } \\
\text { fibroblasts }\end{array}$ \\
\hline BSK_SAg_CD69_down & 0.99 & CD69 & binding reporter & Bioseek & $\begin{array}{l}\text { umbilical vein } \\
\text { endothelium and } \\
\text { peripheral blood } \\
\text { mononuclear } \\
\text { cells }\end{array}$ \\
\hline ATG_PPARg_TRANS_up & 0.99 & PPARG & inducible reporter & Attagene & HepG2 \\
\hline
\end{tabular}




\begin{tabular}{|c|c|c|c|c|c|}
\hline TOX21_FXR_BLA_agonist_ch2 & 1.00 & NR1H4 & background reporter & Tox21/NCGC & HEK293T \\
\hline ATG_RXRa_TRANS_up & 1.00 & RXRA & inducible reporter & Attagene & HepG2 \\
\hline BSK_3C_HLADR_down & 1.00 & HLA-DRA & binding reporter & Bioseek & $\begin{array}{l}\text { umbilical vein } \\
\text { endothelium }\end{array}$ \\
\hline BSK_KF3CT_MCP1_down & 1.00 & CCL2 & binding reporter & Bioseek & $\begin{array}{l}\text { keratinocytes } \\
\text { and foreskin } \\
\text { fibroblasts }\end{array}$ \\
\hline NVS_ADME_rCYP2D2 & 1.01 & Cyp2d2 & enzyme reporter & Novascreen & NA \\
\hline BSK_CASM3C_Proliferation_down & 1.01 & NA & viability reporter & Bioseek & $\begin{array}{l}\text { coronary artery } \\
\text { smooth muscle } \\
\text { cells }\end{array}$ \\
\hline BSK_4H_SRB_down & 1.02 & NA & viability reporter & Bioseek & $\begin{array}{l}\text { umbilical vein } \\
\text { endothelium }\end{array}$ \\
\hline BSK_SAg_MIG_down & 1.02 & CXCL9 & binding reporter & Bioseek & $\begin{array}{l}\text { umbilical vein } \\
\text { endothelium and }\end{array}$ \\
\hline
\end{tabular}


peripheral blood mononuclear cells

\begin{tabular}{lllll}
\hline BSK_4H_uPAR_down & 1.03 & PLAUR & binding reporter & Bioseek \\
endothelium
\end{tabular}

BSK_LPS_Eselectin_down $\quad$ SELE binding reporter umbilical vein

$1.03 \quad$ peripheral blood mononuclear cells

\begin{tabular}{|c|c|c|c|c|c|}
\hline ATG_DR4_LXR_CIS_dn & 1.03 & NR1H3|NR1H2 & inducible reporter & Attagene & HepG2 \\
\hline BSK_3C_Eselectin_down & 1.03 & SELE & binding reporter & Bioseek & $\begin{array}{l}\text { umbilical vein } \\
\text { endothelium }\end{array}$ \\
\hline BSK_KF3CT_SRB_down & 1.03 & NA & viability reporter & Bioseek & $\begin{array}{l}\text { keratinocytes } \\
\text { and foreskin } \\
\text { fibroblasts }\end{array}$ \\
\hline BSK_4H_Eotaxin3_down & 1.04 & CCL26 & binding reporter & Bioseek & $\begin{array}{l}\text { umbilical vein } \\
\text { endothelium }\end{array}$ \\
\hline TOX21_ARE_BLA_agonist_ratio & 1.04 & NFE2L2 & inducible reporter & Tox21/NCGC & HepG2 \\
\hline BSK LPS TissueFactor_down & 1.05 & F3 & binding reporter & Bioseek & umbilical \\
\hline
\end{tabular}


endothelium and peripheral blood mononuclear

cells

\begin{tabular}{|c|c|c|c|c|c|}
\hline NVS_GPCR_hOpiate_D1 & 1.05 & OPRD1 & binding reporter & Novascreen & NA \\
\hline BSK_SAg_SRB_down & 1.05 & NA & viability reporter & Bioseek & $\begin{array}{l}\text { umbilical vein } \\
\text { endothelium and } \\
\text { peripheral blood } \\
\text { mononuclear } \\
\text { cells }\end{array}$ \\
\hline BSK_LPS_MCSF_down & 1.05 & CSF1 & binding reporter & Bioseek & $\begin{array}{l}\text { umbilical vein } \\
\text { endothelium and } \\
\text { peripheral blood } \\
\text { mononuclear } \\
\text { cells }\end{array}$ \\
\hline
\end{tabular}

\begin{tabular}{|c|c|c|c|c|c|}
\hline APR_HepG2_CellLoss_24h_dn & 1.06 & NA & viability reporter & Apredica & HepG2 \\
\hline BSK_3C_VCAM1_down & 1.06 & VCAM1 & binding reporter & Bioseek & $\begin{array}{l}\text { umbilical vein } \\
\text { endothelium }\end{array}$ \\
\hline BSK_LPS_MCP1_down & 1.06 & CCL2 & binding reporter & Bioseek & $\begin{array}{l}\text { umbilical vein } \\
\text { endothelium and } \\
\text { peripheral blood } \\
\text { mononuclear }\end{array}$ \\
\hline
\end{tabular}




\begin{tabular}{|c|c|c|c|c|c|}
\hline APR_HepG2_p53Act_72h_up & 1.07 & TP53 & viability reporter & Apredica & HepG2 \\
\hline BSK_CASM3C_TissueFactor_down & 1.08 & F3 & binding reporter & Bioseek & $\begin{array}{l}\text { coronary artery } \\
\text { smooth muscle } \\
\text { cells }\end{array}$ \\
\hline
\end{tabular}

\begin{tabular}{|c|c|c|c|c|c|}
\hline BSK_LPS_SRB_down & 1.08 & NA & viability reporter & Bioseek & $\begin{array}{l}\text { umbilical vein } \\
\text { endothelium and } \\
\text { peripheral blood } \\
\text { mononuclear } \\
\text { cells }\end{array}$ \\
\hline BSK_BE3C_PAI1_down & 1.08 & SERPINE1 & binding reporter & Bioseek & $\begin{array}{l}\text { bronchial } \\
\text { epithelial cells }\end{array}$ \\
\hline BSK_BE3C_tPA_down & 1.08 & PLAT & binding reporter & Bioseek & $\begin{array}{l}\text { bronchial } \\
\text { epithelial cells }\end{array}$ \\
\hline BSK_BE3C_TGFb1_down & 1.08 & TGFB1 & binding reporter & Bioseek & $\begin{array}{l}\text { bronchial } \\
\text { epithelial cells }\end{array}$ \\
\hline BSK_LPS_IL8_down & 1.08 & CXCL8 & binding reporter & Bioseek & $\begin{array}{l}\text { umbilical vein } \\
\text { endothelium and } \\
\text { peripheral blood } \\
\text { mononuclear }\end{array}$ \\
\hline
\end{tabular}




\begin{tabular}{|c|c|c|c|c|c|}
\hline BSK_CASM3C_uPAR_down & 1.09 & PLAUR & binding reporter & Bioseek & $\begin{array}{l}\text { coronary artery } \\
\text { smooth muscle } \\
\text { cells }\end{array}$ \\
\hline BSK_KF3CT_IL1a_down & 1.09 & IL1A & binding reporter & Bioseek & $\begin{array}{l}\text { keratinocytes } \\
\text { and foreskin } \\
\text { fibroblasts }\end{array}$ \\
\hline BSK_BE3C_IP10_down & 1.10 & CXCL10 & binding reporter & Bioseek & $\begin{array}{l}\text { bronchial } \\
\text { epithelial cells }\end{array}$ \\
\hline BSK_3C_IL8_down & 1.10 & CXCL8 & binding reporter & Bioseek & $\begin{array}{l}\text { umbilical vein } \\
\text { endothelium }\end{array}$ \\
\hline NVS_TR_rNET & 1.10 & Slc6a2 & binding reporter & Novascreen & NA \\
\hline BSK_hDFCGF_IL8_down & 1.10 & CXCL8 & binding reporter & Bioseek & $\begin{array}{l}\text { foreskin } \\
\text { fibroblast }\end{array}$ \\
\hline BSK_BE3C_uPAR_down & 1.10 & PLAUR & binding reporter & Bioseek & $\begin{array}{l}\text { bronchial } \\
\text { epithelial cells }\end{array}$ \\
\hline BSK_KF3CT_IP10_down & 1.11 & CXCL10 & binding reporter & Bioseek & $\begin{array}{l}\text { keratinocytes } \\
\text { and foreskin } \\
\text { fibroblasts }\end{array}$ \\
\hline
\end{tabular}




\begin{tabular}{|c|c|c|c|c|c|}
\hline ACEA_T47D_80hr_Negative & 1.11 & NA & growth reporter & $\begin{array}{l}\text { ACEA } \\
\text { Biosciences }\end{array}$ & $\mathrm{T} 47 \mathrm{D}$ \\
\hline BSK_4H_VCAM1_down & 1.12 & VCAM1 & binding reporter & Bioseek & $\begin{array}{l}\text { umbilical vein } \\
\text { endothelium }\end{array}$ \\
\hline BSK_LPS_VCAM1_down & 1.12 & VCAM1 & binding reporter & Bioseek & $\begin{array}{l}\text { umbilical vein } \\
\text { endothelium and } \\
\text { peripheral blood } \\
\text { mononuclear } \\
\text { cells }\end{array}$ \\
\hline NVS_ADME_rCYP2C13 & 1.12 & Сyp2c13 & enzyme reporter & Novascreen & NA \\
\hline BSK_KF3CT_uPA_down & 1.12 & PLAU & binding reporter & Bioseek & $\begin{array}{l}\text { keratinocytes } \\
\text { and foreskin } \\
\text { fibroblasts }\end{array}$ \\
\hline BSK_3C_MCP1_down & 1.13 & CCL2 & binding reporter & Bioseek & $\begin{array}{l}\text { umbilical vein } \\
\text { endothelium }\end{array}$ \\
\hline BSK_CASM3C_LDLR_down & 1.14 & LDLR & binding reporter & Bioseek & $\begin{array}{l}\text { coronary artery } \\
\text { smooth muscle } \\
\text { cells }\end{array}$ \\
\hline APR_Hepat_DNADamage_1hr_dn & 1.14 & TP53 & viability reporter & Apredica & hepatocyte \\
\hline BSK_BE3C_MMP1_down & 1.14 & MMP1 & binding reporter & Bioseek & bronchial \\
\hline
\end{tabular}


epithelial cells

BSK_CASM3C_HLADR_down

HLA-DRA

binding reporter

Bioseek

coronary artery

1.14 cells

\begin{tabular}{lcccc}
\hline NVS_NR_hPXR & 1.14 & NR1I2 & binding reporter & Novascreen \\
\hline BSK_BE3C_HLADR_down & 1.15 & HLA-DRA & binding reporter & $\begin{array}{c}\text { Bioseek } \\
\text { bronchial } \\
\text { epithelial cells }\end{array}$ \\
\hline BSK_3C_ICAM1_down & & binding reporter & Bioseek & $\begin{array}{l}\text { umbilical vein } \\
\text { endothelium }\end{array}$
\end{tabular}

\begin{tabular}{|c|c|c|c|c|c|}
\hline OT_FXR_FXRSRC1_0480 & 1.15 & NR1H4|SRC & binding reporter & Odyssey Thera & HEK293T \\
\hline BSK_CASM3C_SRB_down & 1.16 & NA & viability reporter & Bioseek & $\begin{array}{l}\text { coronary artery } \\
\text { smooth muscle } \\
\text { cells }\end{array}$ \\
\hline
\end{tabular}

BSK_LPS_TNFa_down

TNF

binding reporter

Bioseek

umbilical vein

endothelium and

1.16

peripheral blood

mononuclear

cells

1.16

CSF1

binding reporter

Bioseek

coronary artery

smooth muscle 

cells

BSK_CASM3C_SAA_down

SAA1

binding reporter

Bioseek

coronary artery

1.17

smooth muscle

cells

\begin{tabular}{|c|c|c|c|c|c|}
\hline TOX21_ARE_BLA_Agonist_ch2 & 1.17 & NFE2L2 & background reporter & Tox21/NCGC & HepG2 \\
\hline BSK_3C_MIG_down & 1.17 & CXCL9 & binding reporter & Bioseek & $\begin{array}{l}\text { umbilical vein } \\
\text { endothelium }\end{array}$ \\
\hline TOX21_AR_LUC_MDAKB2_Antagonist2 & 1.18 & NA & inducible reporter & Tox21/NCGC & NA \\
\hline BSK_BE3C_SRB_down & 1.19 & NA & viability reporter & Bioseek & $\begin{array}{l}\text { bronchial } \\
\text { epithelial cells }\end{array}$ \\
\hline NVS_TR_hDAT & 1.19 & SLC6A3 & binding reporter & Novascreen & NA \\
\hline BSK_BE3C_uPA_down & 1.21 & PLAU & binding reporter & Bioseek & $\begin{array}{l}\text { bronchial } \\
\text { epithelial cells }\end{array}$ \\
\hline BSK_KF3CT_TIMP2_down & 1.21 & TIMP2 & binding reporter & Bioseek & $\begin{array}{l}\text { keratinocytes } \\
\text { and foreskin } \\
\text { fibroblasts }\end{array}$ \\
\hline
\end{tabular}




\begin{tabular}{|c|c|c|c|c|c|}
\hline BSK_BE3C_MIG_down & 1.21 & CXCL9 & binding reporter & Bioseek & $\begin{array}{l}\text { bronchial } \\
\text { epithelial cells }\end{array}$ \\
\hline BSK_KF3CT_ICAM1_down & 1.21 & ICAM1 & binding reporter & Bioseek & $\begin{array}{l}\text { keratinocytes } \\
\text { and foreskin } \\
\text { fibroblasts }\end{array}$ \\
\hline NVS_NR_cAR & 1.22 & AR & binding reporter & Novascreen & NA \\
\hline NVS_ENZ_hMMP7 & 1.24 & MMP7 & enzyme reporter & Novascreen & NA \\
\hline NVS_GPCR_hOpiate_mu & 1.28 & OPRM1 & binding reporter & Novascreen & NA \\
\hline APR_Hepat_Apoptosis_1hr_dn & 1.31 & CYCS & morphology reporter & Apredica & hepatocyte \\
\hline TOX21_AR_BLA_Antagonist_viability & 1.34 & NA & viability reporter & Tox21/NCGC & HEK293T \\
\hline NVS_GPCR_rSST & 1.35 & Sstr 1 & binding reporter & Novascreen & NA \\
\hline NVS_ENZ_oCOX2 & 1.37 & PTGS2 & enzyme reporter & Novascreen & NA \\
\hline BSK_BE3C_IL1a_down & 1.38 & IL1A & binding reporter & Bioseek & $\begin{array}{l}\text { bronchial } \\
\text { epithelial cells }\end{array}$ \\
\hline TOX21_Aromatase_Inhibition & 1.39 & CYP19A1 & inducible reporter & Tox21/NCGC & $\mathrm{MCF}-7$ \\
\hline APR_Hepat_CellLoss_1hr_dn & 1.40 & NA & viability reporter & Apredica & hepatocyte \\
\hline NCCT_QuantiLum_inhib_dn & 1.40 & NA & enzyme reporter & NCCT's & NA \\
\hline
\end{tabular}


Simmons Lab

\begin{tabular}{|c|c|c|c|c|c|}
\hline TOX21_AR_BLA_Antagonist_ch2 & 1.40 & AR & background reporter & Tox21/NCGC & HEK293T \\
\hline NVS_NR_rAR & 1.40 & $\mathrm{Ar}$ & binding reporter & Novascreen & NA \\
\hline Tanguay_ZF_120hpf_MORT_up & 1.41 & NA & NA & Tanguay Lab & $\begin{array}{l}\text { dechorionated } \\
\text { zebrafish } \\
\text { embryo }\end{array}$ \\
\hline
\end{tabular}

\begin{tabular}{|c|c|c|c|c|c|}
\hline NVS_NR_bPR & 1.41 & PGR & binding reporter & Novascreen & NA \\
\hline NVS_TR_gDAT & 1.42 & Slc6a3 & binding reporter & Novascreen & NA \\
\hline TOX21_GR_BLA_Agonist_ch1 & 1.46 & NR3C1 & background reporter & Tox $21 /$ NCGC & $\mathrm{HeLa}$ \\
\hline NVS_ENZ_rMAOAC & 1.46 & Maoa & enzyme reporter & Novascreen & NA \\
\hline TOX21_HSE_BLA_agonist_ch1 & 1.47 & HSF1 & background reporter & Tox21/NCGC & HeLa \\
\hline NVS_GPCR_mCCKAPeripheral & 1.48 & Cckar & binding reporter & Novascreen & NA \\
\hline APR_HepG2_MitoticArrest_72h_up & 1.48 & $\mathrm{H} 3 \mathrm{~F} 3 \mathrm{~A}$ & morphology reporter & Apredica & HepG2 \\
\hline TOX21_AR_BLA_Antagonist_ratio & 1.48 & $\mathrm{AR}$ & inducible reporter & Tox21/NCGC & HEK293T \\
\hline APR_HepG2_MitoMass_24h_dn & 1.49 & NA & morphology reporter & Apredica & HepG2 \\
\hline APR_HepG2_OxidativeStress_72h_up & 1.50 & $\mathrm{H} 2 \mathrm{AFX}$ & viability reporter & Apredica & HepG2 \\
\hline
\end{tabular}




\begin{tabular}{|c|c|c|c|c|c|}
\hline TOX21_AR_LUC_MDAKB2_Antagonist & 1.51 & $\mathrm{AR}$ & inducible reporter & Tox21/NCGC & MDA-kb2 \\
\hline ATG_XTT_Cytotoxicity_up & 1.51 & NA & viability reporter & Attagene & HepG2 \\
\hline TOX21_p53_BLA_p1_viability & 1.51 & NA & viability reporter & Tox21/NCGC & HCT116 \\
\hline BSK_CASM3C_Thrombomodulin_down & 1.52 & THBD & binding reporter & Bioseek & $\begin{array}{l}\text { coronary artery } \\
\text { smooth muscle } \\
\text { cells }\end{array}$ \\
\hline BSK_CASM3C_VCAM1_down & 1.52 & VCAM1 & binding reporter & Bioseek & $\begin{array}{l}\text { coronary artery } \\
\text { smooth muscle } \\
\text { cells }\end{array}$ \\
\hline BSK_CASM3C_IL8_down & 1.52 & CXCL8 & binding reporter & Bioseek & $\begin{array}{l}\text { coronary artery } \\
\text { smooth muscle } \\
\text { cells }\end{array}$ \\
\hline BSK_CASM3C_MIG_down & 1.52 & CXCL9 & binding reporter & Bioseek & $\begin{array}{l}\text { coronary artery } \\
\text { smooth muscle } \\
\text { cells }\end{array}$ \\
\hline NVS_GPCR_hNK2 & 1.53 & TACR2 & binding reporter & Novascreen & NA \\
\hline APR_HepG2_OxidativeStress_24h_up & 1.53 & H2AFX & viability reporter & Apredica & HepG2 \\
\hline NVS_GPCR_hAdrb1 & 1.54 & ADRB1 & binding reporter & Novascreen & NA \\
\hline
\end{tabular}




\begin{tabular}{|c|c|c|c|c|c|}
\hline TOX21_ERa_BLA_Antagonist_ratio & 1.54 & ESR1 & inducible reporter & Tox21/NCGC & HEK293T \\
\hline APR_HepG2_p53Act_24h_up & 1.54 & TP53 & viability reporter & Apredica & HepG2 \\
\hline APR_HepG2_CellLoss_72h_dn & 1.54 & NA & viability reporter & Apredica & HepG2 \\
\hline NVS_GPCR_h5HT7 & 1.55 & HTR7 & binding reporter & Novascreen & NA \\
\hline TOX21_HSE_BLA_agonist_ratio & 1.56 & HSF1 & inducible reporter & Tox $21 /$ NCGC & $\mathrm{HeLa}$ \\
\hline TOX21_NFkB_BLA_agonist_viability & 1.56 & NA & viability reporter & Tox21/NCGC & ME-180 \\
\hline TOX21_PPARd_BLA_Agonist_viability & 1.56 & NA & viability reporter & Tox21/NCGC & HEK293T \\
\hline TOX21_NFkB_BLA_agonist_ch1 & 1.57 & NFKB1 & background reporter & Tox21/NCGC & ME-180 \\
\hline TOX21_p53_BLA_p1_ch1 & 1.57 & TP53 & background reporter & Tox21/NCGC & HCT116 \\
\hline TOX21_GR_BLA_Antagonist_ch2 & 1.59 & $\mathrm{NR} 3 \mathrm{C} 1$ & background reporter & Tox21/NCGC & $\mathrm{HeLa}$ \\
\hline TOX21_ERa_BLA_Antagonist_viability & 1.59 & NA & viability reporter & Tox21/NCGC & HEK293T \\
\hline NVS_ENZ_rMAOBP & 1.61 & Maob & enzyme reporter & Novascreen & NA \\
\hline NVS_ENZ_hAKT2 & 1.61 & AKT2 & enzyme reporter & Novascreen & NA \\
\hline TOX21_GR_BLA_Agonist_ratio & 1.61 & $\mathrm{NR} 3 \mathrm{C} 1$ & inducible reporter & Tox21/NCGC & $\mathrm{HeLa}$ \\
\hline TOX21_VDR_BLA_antagonist_viability & 1.62 & NA & viability reporter & Tox21/NCGC & HEK293T \\
\hline
\end{tabular}




\begin{tabular}{|c|c|c|c|c|c|}
\hline TOX21_ESRE_BLA_ch1 & 1.62 & ATF6 & background reporter & Tox21/NCGC & HeLa \\
\hline Tanguay_ZF_120hpf_ActivityScore & 1.63 & NA & NA & Tanguay Lab & $\begin{array}{l}\text { dechorionated } \\
\text { zebrafish } \\
\text { embryo }\end{array}$ \\
\hline TOX21_p53_BLA_p4_viability & 1.63 & NA & viability reporter & Tox21/NCGC & HCT116 \\
\hline TOX21_p53_BLA_p2_viability & 1.63 & NA & viability reporter & Tox21/NCGC & НCT116 \\
\hline TOX21_p53_BLA_p5_viability & 1.65 & NA & viability reporter & Tox21/NCGC & HCT116 \\
\hline TOX21_ESRE_BLA_ratio & 1.66 & ATF6 & inducible reporter & Tox21/NCGC & HeLa \\
\hline TOX21_p53_BLA_p4_ch1 & 1.66 & TP53 & background reporter & Tox21/NCGC & HCT116 \\
\hline APR_Hepat_MitoFxnI_1hr_dn & 1.67 & NA & membrane potential reporter & Apredica & hepatocyte \\
\hline TOX21_p53_BLA_p2_ch1 & 1.67 & TP53 & background reporter & Tox21/NCGC & НCТ116 \\
\hline NCCT_TPO_AUR_dn & 1.68 & Тро & enzyme reporter & $\begin{array}{l}\text { NCCT's } \\
\text { Simmons Lab }\end{array}$ & NA \\
\hline APR_HepG2_StressKinase_72h_up & 1.68 & JUN & enzyme reporter & Apredica & HepG2 \\
\hline TOX21_FXR_BLA_antagonist_ratio & 1.69 & NR1H4 & inducible reporter & Tox21/NCGC & HEK293T \\
\hline TOX21_GR_BLA_Antagonist_viability & 1.69 & NA & viability reporter & Tox21/NCGC & HeLa \\
\hline
\end{tabular}




\begin{tabular}{|c|c|c|c|c|c|}
\hline TOX21_HSE_BLA_agonist_viability & 1.69 & NA & viability reporter & Tox21/NCGC & HeLa \\
\hline TOX21_p53_BLA_p5_ch1 & 1.77 & TP53 & background reporter & Tox21/NCGC & HCT116 \\
\hline TOX21_p53_BLA_p3_viability & 1.78 & NA & viability reporter & Tox21/NCGC & HCT116 \\
\hline TOX21_p53_BLA_p3_ch1 & 1.79 & TP53 & background reporter & Tox21/NCGC & HCT116 \\
\hline APR_HepG2_NuclearSize_24h_dn & 1.80 & NA & morphology reporter & Apredica & HepG2 \\
\hline TOX21_FXR_BLA_antagonist_viability & 1.81 & NA & viability reporter & Tox21/NCGC & HEK293T \\
\hline APR_HepG2_MitoticArrest_24h_up & 1.81 & H3F3A & morphology reporter & Apredica & HepG2 \\
\hline APR_Hepat_CellLoss_24hr_dn & 1.82 & NA & viability reporter & Apredica & hepatocyte \\
\hline TOX21_VDR_BLA_Agonist_viability & 1.84 & NA & viability reporter & Tox21/NCGC & HEK293T \\
\hline APR_HepG2_MicrotubuleCSK_72h_dn & 1.85 & TUBA1A & conformation reporter & Apredica & HepG2 \\
\hline TOX21_ERa_LUC_BG1_Antagonist & 1.85 & ESR1 & inducible reporter & Tox21/NCGC & BG1 \\
\hline APR_Hepat_DNATexture_48hr_up & 1.85 & NA & conformation reporter & Apredica & hepatocyte \\
\hline APR_Hepat_CellLoss_48hr_dn & 1.86 & NA & viability reporter & Apredica & hepatocyte \\
\hline TOX21_p53_BLA_p1_ratio & 1.86 & TP53 & inducible reporter & Tox21/NCGC & HCT116 \\
\hline APR Hepat DNADamage $48 \mathrm{hr}$ up & 1.87 & TP53 & viability reporter & Apredica & hepatocyte \\
\hline
\end{tabular}




\begin{tabular}{|c|c|c|c|c|c|}
\hline APR_Hepat_Apoptosis_48hr_up & 1.87 & CYCS & morphology reporter & Apredica & hepatocyte \\
\hline APR_Hepat_Steatosis_48hr_up & 1.88 & NA & morphology reporter & Apredica & hepatocyte \\
\hline APR_Hepat_DNATexture_24hr_up & 1.88 & NA & conformation reporter & Apredica & hepatocyte \\
\hline TOX21_p53_BLA_p5_ratio & 1.89 & TP53 & inducible reporter & Tox21/NCGC & HCT116 \\
\hline APR_Hepat_NuclearSize_48hr_dn & 1.89 & NA & morphology reporter & Apredica & hepatocyte \\
\hline APR_Hepat_Apoptosis_24hr_up & 1.90 & CYCS & morphology reporter & Apredica & hepatocyte \\
\hline APR_Hepat_DNADamage_24hr_up & 1.91 & TP53 & viability reporter & Apredica & hepatocyte \\
\hline TOX21_p53_BLA_p4_ratio & 1.91 & TP53 & inducible reporter & Tox21/NCGC & HCT116 \\
\hline TOX21_p53_BLA_p3_ratio & 1.91 & TP53 & inducible reporter & Tox21/NCGC & HCT116 \\
\hline TOX21_AR_BLA_Agonist_ratio & 1.93 & AR & inducible reporter & Tox21/NCGC & HEK293T \\
\hline APR_Hepat_NuclearSize_24hr_dn & 1.94 & NA & morphology reporter & Apredica & hepatocyte \\
\hline APR_Hepat_MitoFxnI_48hr_dn & 1.97 & NA & membrane potential reporter & Apredica & hepatocyte \\
\hline TOX21_ARE_BLA_Agonist_ch1 & 2.00 & NFE2L2 & background reporter & Tox21/NCGC & HepG2 \\
\hline APR_Hepat_Steatosis_1hr_dn & 2.01 & NA & morphology reporter & Apredica & hepatocyte \\
\hline APR_HepG2_OxidativeStress_1h_up & 2.04 & H2AFX & viability reporter & Apredica & HepG2 \\
\hline
\end{tabular}




\begin{tabular}{|c|c|c|c|c|c|}
\hline APR_HepG2_NuclearSize_1h_dn & 2.04 & NA & morphology reporter & Apredica & HepG2 \\
\hline TOX21_p53_BLA_p2_ratio & 2.05 & TP53 & inducible reporter & Tox21/NCGC & HCT116 \\
\hline APR_HepG2_MitoMass_1h_dn & 2.06 & NA & morphology reporter & Apredica & HepG2 \\
\hline APR_HepG2_CellCycleArrest_1h_dn & 2.07 & NA & morphology reporter & Apredica & HepG2 \\
\hline APR_Hepat_MitoFxnI_24hr_dn & 2.08 & NA & membrane potential reporter & Apredica & hepatocyte \\
\hline APR_HepG2_CellLoss_1h_dn & 2.09 & NA & viability reporter & Apredica & HepG2 \\
\hline TOX21_ESRE_BLA_viability & 2.26 & NA & viability reporter & Tox21/NCGC & $\mathrm{HeLa}$ \\
\hline TOX21_NFkB_BLA_agonist_ratio & 2.40 & NFKB1 & inducible reporter & Tox21/NCGC & ME-180 \\
\hline
\end{tabular}

*The rows in grey color are 33 selected "gene-based HepG2" assays 
Table S15. Six overlapped genes between ToxCast responsive genes and CRISPR-Cas 9 resistant/sensitive genes of triclosan

\begin{tabular}{|c|c|c|c|}
\hline Gene Symbols & CRISPR-Cas9 resistant/sensitive genes & Obesity & Breast cancer \\
\hline NR1I2 & IC50 Sensitive Genes & $\mathrm{Y}$ & $\mathrm{Y}$ \\
\hline PAX6 & IC50 Resistant Genes & $\mathrm{Y}$ & $\mathrm{N}$ \\
\hline XBP1 & IC20 Resistant Genes & $\mathrm{Y}$ & $\mathrm{Y}$ \\
\hline SP1 & IC50 Sensitive Genes & $\mathrm{N}$ & $\mathrm{Y}$ \\
\hline PPARG & IC50 Resistant Genes & $\mathrm{Y}$ & $\mathrm{Y}$ \\
\hline NR1H3 & IC50 Sensitive Genes & $\mathrm{Y}$ & $\mathrm{N}$ \\
\hline
\end{tabular}

Y: The gene may be potentially associated with the disease term according to DisGeNET database

$\mathrm{N}$ : The gene may not be potentially associated with the disease term according to DisGeNET database 